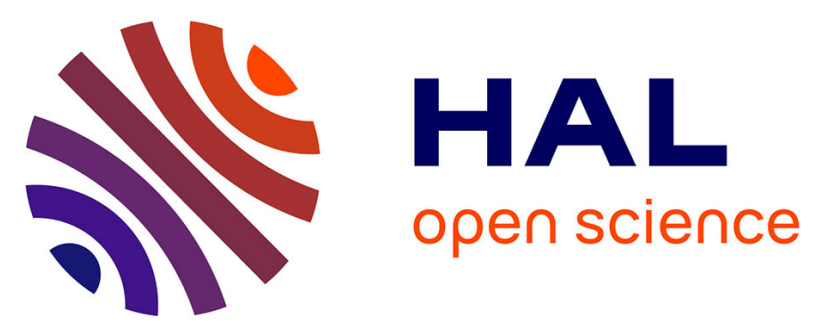

\title{
Polar N-terminal Residues Conserved in Type 2 Secretion Pseudopilins Determine Subunit Targeting and Membrane Extraction Steps during Fibre Assembly
}

Javier Santos-Moreno, Alexandra East, Ingrid Guilvout, Nathalie Nadeau, Peter J. Bond, Guy Tran van Nhieu, Olivera Francetic

\section{To cite this version:}

Javier Santos-Moreno, Alexandra East, Ingrid Guilvout, Nathalie Nadeau, Peter J. Bond, et al.. Polar N-terminal Residues Conserved in Type 2 Secretion Pseudopilins Determine Subunit Targeting and Membrane Extraction Steps during Fibre Assembly. Journal of Molecular Biology, 2017, 429 (11), pp.1746 - 1765. 10.1016/j.jmb.2017.04.005 . pasteur-01602354

\section{HAL Id: pasteur-01602354}

https://hal-pasteur.archives-ouvertes.fr/pasteur-01602354

Submitted on 2 Oct 2017

HAL is a multi-disciplinary open access archive for the deposit and dissemination of scientific research documents, whether they are published or not. The documents may come from teaching and research institutions in France or abroad, or from public or private research centers.
L'archive ouverte pluridisciplinaire HAL, est destinée au dépôt et à la diffusion de documents scientifiques de niveau recherche, publiés ou non, émanant des établissements d'enseignement et de recherche français ou étrangers, des laboratoires publics ou privés. 
1 Polar N-terminal residues conserved in type 2 secretion pseudopilins determine subunit

2 targeting and membrane extraction steps during fibre assembly

3

4 Javier Santos-Moreno $^{1-5 \#}$, Alexandra East ${ }^{6}$, Ingrid Guilvout ${ }^{7,8}$, Nathalie Nadeau ${ }^{7}$, Peter J.

5 Bond $^{9,10}$, Guy Tran Van Nhieu ${ }^{2-5}$ and Olivera Francetic ${ }^{7,8^{*}}$

6

$7 \quad{ }^{1}$ Université Paris Diderot (Paris 7) Sorbonne Paris Cité

$8{ }^{2}$ Laboratory of Intercellular Communication and Microbial Infections, CIRB, Collège de

9 France, Paris, France

$10{ }^{3}$ Institut National de la Santé et de la Recherche Médicale (Inserm) U1050, France

$11{ }^{4}$ Centre National de la Recherche Scientifique (CNRS), UMR7241, France

$12{ }^{5}$ MEMOLIFE Laboratory of Excellence and Paris Sciences et Lettres, France

$13{ }^{6}$ Centre for Molecular Informatics, Department of Chemistry, University of Cambridge,

14 Lensfield Road, Cambridge, CB2 1EW, UK

$15{ }^{7}$ Laboratory of Macromolecular Systems and Signalling, Institut Pasteur, Department of

16 Microbiology and CNRS ERL6002, 25 rue du Dr Roux, 75724 Paris, Cedex 15, France

$17{ }^{8}$ Biochemistry of Macromolecular Interactions Unit, Department of Structural Biology and

18 Chemistry, 28 rue du Dr Roux, 75724 Paris, Cedex 15, France

$19{ }^{9}$ Bioinformatics Institute (A*STAR), 30 Biopolis Str, \#07-01 Matrix, Singapore 138671

$20{ }^{10}$ National University of Singapore, Department of Biological Sciences, 14 Science Drive 4,

21 Singapore 117543

$23{ }^{\#}$ Current address: Department of Fundamental Microbiology, University of Lausanne,

24 Biophore building, CH1015 Lausanne, Switzerland.

$26{ }^{*}$ Correspondence:

27 Institut Pasteur, 25 rue du Dr Roux, 75724 Paris CEDEX 15, France

28 email: ofrancet@pasteur.fr

29 Tel: 33140613681

30 Fax: +3314568 8960

31 Key words: type 4 fibre assembly, membrane proteins, immunofluorescence microscopy,

32 molecular dynamics simulations, N-methylation 


\section{Abstract}

36 Bacterial type 2 secretion systems (T2SS), type 4 pili (T4P) and archaeal flagella assemble

37 fibres from initially membrane-embedded pseudopilin and pilin subunits. Fibre subunits are 38 made as precursors with positively charged N-terminal anchors, whose cleavage via the 39 prepilin peptidase, essential for pilin membrane extraction and assembly, is followed by N40 methylation of the mature (pseudo)pilin N-terminus. The conserved Glu residue at position 5 41 (E5) of mature (pseudo)pilins is essential for assembly. Unlike T4 pilins, where E5 residue

42 substitutions also abolish N-methylation, the E5A variant of T2SS pseudopilin PulG remains $43 \mathrm{~N}$-methylated, but is affected in interaction with the T2SS component PulM. Here, 44 biochemical and functional analyses showed that the PulM interaction defect only partly 45 accounts for the $\mathrm{PulG}^{\mathrm{E} 5 \mathrm{~A}}$ assembly defect. First, PulG ${ }^{\mathrm{T} 2 \mathrm{~A}}$ variant, equally defective in PulM 46 interaction, remained partially functional. Furthermore, pseudopilus assembly defect of

47 pulG(E5A) mutant was stronger than that of the pulM deletion mutant. To understand the 48 dominant effect of E5A mutation, we used molecular dynamics simulations of PulG ${ }^{\mathrm{E} \text { A }}$, 49 methylated $\mathrm{PulG}^{\mathrm{WT}}\left(\mathrm{MePulG}^{\mathrm{WT}}\right)$ and $\mathrm{MePulG}^{\mathrm{E} 5 \mathrm{~A}}$ variant in a model membrane. These 50 simulations pointed to a key role for an intra-molecular interaction between the pseudopilin 51 N-terminal amine and E5 to limit polar interactions with membrane phospholipids. N-

52 methylation of the N-terminal amine further limited it interactions with phospholipid head53 groups to facilitate pseudopilin membrane escape. By binding to polar residues in the 54 conserved $\mathrm{N}$-terminal region of $\mathrm{PulG}$, we propose that PulM acts as chaperone to promote 55 pseudopilin recruitment and coordinate its membrane extraction with subsequent steps of the 56 fibre assembly process. 


\section{Introduction}

60

61 Prokaryotes build diverse surface appendages and protein transport systems to colonize their

62 niche and acquire nutrients. Some of the most ancient and versatile prokaryotic nanomachines

63 that mediate these functions belong to the type 4 filament (Tff) superfamily ${ }^{1}$. These

64 conserved membrane complexes use the ATP-derived energy to drive assembly of flagella

65 (archaella) and pili in archaea ${ }^{2 ; 3 ; 4}$ and to build T4P and T2SS pseudopili in bacteria.

66 Illustrating the diversity of Tff functions, T4P, thin bacterial surface fibres, mediate

67 adherence, aggregation, motility, protein transport and DNA uptake ${ }^{1 ; 5}$. In T2SSs, found in

68 Gram-negative (or diderm) bacteria, short periplasmic pseudopilus fibres promote protein

69 transport from the periplasm across the outer membrane $(\mathrm{OM})^{6 ; 7 ; 8}$.

70 Bacterial Tffs are helical polymers of repeating subunits of the major pilin or

71 pseudopilin, which may also contain one or more minor subunits that modulate the fibre

72 assembly and function ${ }^{1}$. These subunits are made as membrane-embedded precursors, with

73 an N-terminal cytoplasmic pre-peptide, followed by a highly conserved transmembrane

74 segment (TMS) and a variable periplasmic globular domain that determines surface features

75 of assembled fibres. The prepilin peptidase, an integral membrane aspartic protease, cleaves

76 the positively charged peptide anchor on the cytoplasmic face of the TMS ${ }^{9}$. Bacterial prepilin

77 peptidases have an additional, methyl-transferase domain that modifies the N-terminus of

78 mature (pseudo)pilins; however the function of this N-methylation remains unclear ${ }^{10 ; 11}$.

79 In Gram-negative bacteria, Tff assembly systems form large envelope-spanning

80 complexes that have been visualised recently for T4P by cryo-electron tomography ${ }^{12}$. Their

81 most conserved parts are the cytoplasmic hexameric ATPase of the GspE family and the inner

82 membrane (IM) platform protein of the GspF family ${ }^{13}$. Together with GspL and GspM, they 
83 form the assembly platform (AP) ${ }^{14}$, which connects via the IM protein GspC with the GspD

84 multimer forming the outer membrane secretin channel ${ }^{15}$.

85 In the T2SS of Klebsiella, dedicated to secretion of pullulanase (PulA),

86 overproduction of the major pseudopilin PulG leads to assembly of fibres on the cell surface

87 in plate-grown bacteria ${ }^{16}$. A similar phenomenon has been observed for the Pseudomonas

88 T2SSs called Xcp ${ }^{17}$. Fibre assembly in overexpression conditions globally correlates with the

89 ability of these systems to promote protein transport under physiological conditions,

90 suggesting a mechanistic link between these two functions ${ }^{16}$. Some interactions of the T2SS

91 major pseudopilin with assembly components have been recently unveiled. The PulG

92 homologue in Vibrio cholerae, EpsG, forms a cross-linked heterodimer with EpsL, even in

93 the absence of other Eps components ${ }^{18}$. EpsL is a bitopic IM protein that binds the ATPase

94 EpsE via its cytoplasmic domain ${ }^{19 ; 20 ; 21}$ and has been proposed to couple ATP hydrolysis to

95 major pseudopilin polymerization ${ }^{18}$. Direct interaction of PulL and PulM T2SS homologues

96 in different bacteria leads to their mutual stabilisation $19 ; 22 ; 23 ; 24$. Recent studies using

97 bacterial two-hybrid $(\mathrm{BAC} 2 \mathrm{H})$ and co-purification approaches have shown that PulG interacts

98 individually with PulM and PulF components of the inner membrane assembly platform ${ }^{25}$.

99 Binding of major pilins to PulF, PulL and PulM homologues has also been demonstrated in 100 the T4P assembly system of Neisseria meningitidis ${ }^{26}$, Thermus thermophilus ${ }^{27}$ and

101 Pseudomonas aeruginosa ${ }^{28}$, further supporting the direct role of AP in fibre assembly.

102 In the $P$. aeruginosa and $N$. gonorrhoeae major T4 pilin subunits, residue E5 is 103 essential for pilus assembly and N-methylation ${ }^{9 ; 29 ; 30}$. In contrast, PulG variants with E5A or 104 E5V substitutions are still $\mathrm{N}$-methylated ${ }^{25 ;} 31$, nevertheless they are fully deficient in 105 pseudopilus assembly and protein secretion, suggesting that this mutation affects a key step in 106 the process ${ }^{31 ; 32 ; 33 ; 34}$. Recently we showed that residue E5 of PulG is a key determinant of 107 PulG \&PulM interaction in the absence of the other T2SS components ${ }^{25}$. Here, we studied the 
108 role of this interaction and PulM function in the context of the T2SS. Since the defective

109 interaction with PulM could not fully explain the loss of function of $\mathrm{PulG}^{\mathrm{E} 5 \mathrm{~A}}$ variant, we used

110 molecular dynamics (MD) simulations to study PulG interactions with model membranes.

111 The results suggested that both E5 and N-methylation reduce polar interactions of pseudopilin

112 N-terminus with membrane phospholipids, revealing an essential stage of Tff assembly.

\section{Results}

116 PulM requirement for PulA secretion and pseudopilus assembly

118 Recent studies have identified the AP protein PulM as a strong interacting partner of PulG ${ }^{25}$.

119 PulM is a bitopic IM component of the AP that stabilizes PulL, which provides the membrane

120 anchor to the ATPase PulE ${ }^{19 ; 22 ; 23 ; 24}$. Unlike its homologues from Vibrio or Pseudomonas

121 T2SSs, previous studies showed that PulM is only partially required for PulA secretion in

122 strains overexpressing pul genes ${ }^{24}$, although it was essential for assembly of PulG pili ${ }^{16}$.

123 Since in the past the roles of some factors involved in secretion had been overlooked due to

124 overexpression, we reinvestigated the role of PulM in PulA secretion under conditions where

125 pul genes were expressed at near-physiological levels, in Escherichia coli strain PAP5199

126 carrying the $p c n B$ mutation that reduces the copy number of expression plasmids (Materials

127 and Methods).

128 At near-physiological expression levels, virtually all PulA was secreted in a strain

129 producing all Pul T2SS components (Fig. 1A, WT lanes 5, 6; Fig. 1C) and secretion was

130 abolished in the negative control strains lacking the PulE ATPase or PulG (Fig. 1A, lanes 1-4;

131 Fig. 1C). A newly constructed mutant strain carrying a complete pulM deletion (plasmid

132 pCHAP8496, Table 1 and Materials and Methods) had the same phenotype (Fig. 1A, lanes 7, 
133 8; Fig. 1C), showing that PulM is essential for PulA secretion under physiological conditions.

134 As PulM is involved in PulL stabilization, we asked whether function could be restored by co-

135 expressing an extra copy of pulL from a $\mathrm{P}_{\text {lacz }}$ promoter in a compatible plasmid (Fig. 1A,

136 lanes 9, 10; Fig. 1C). However, increased PulL levels did not improve PulA secretion,

137 suggesting that PulM might play an additional role. The secretion defect of the $\Delta p u l M$ mutant

138 was complemented with pulM or pulLM expressed in trans (Fig. 1A, lanes 13-16; Fig. 1C).

139 The Pul T2SS is able to assemble PulG into fibres on the bacterial surface, when the

$140 \mathrm{pul}$ genes are overexpressed from a moderate copy-number plasmid in bacteria cultured on

141 LB agar media ${ }^{16}$. Here, using the new $\Delta p u l M$ allele, we re-assessed the role of PulM in 142 piliation (Materials and Methods) (Fig. 1B). PulG fibres were assembled in a manner fully 143 dependent on PulE ATPase (Fig. 1B, lanes 1, 2 and 5, 6; Fig. 1D), while no signal was 144 observed in the control lacking PulG (Fig. 1B, lanes 3, 4; Fig. 1D). The absence of PulM led 145 to a severe defect, but did not fully abolish piliation (Fig. 1B, lanes 7, 8; Fig. 1D), confirming 146 our previous observations ${ }^{7}$. In contrast, PulL, which recruits PulE to the AP, was essential for 147 piliation (Fig. 1B, lanes 11, 12). Piliation in the $\Delta p u l M$ strain was restored by 148 complementation with pulM (Fig. 1B, lanes 13, 14; Fig. 1D), while overproduction of PulL

149 exacerbated the piliation defects in $\Delta p u l M+p u l L$ and $\Delta p u l M+$ pulLpulM strains (Fig. 1B, 150 compare lanes 7, 8 with 9, 10 and 13, 14 with 15, 16; Fig. 1D), indicating that assembly of 151 surface pili requires the correct ratio between these Pul components. PulM was destabilized in 152 the absence of PulL, and the reverse was also true (Fig. 1B) confirming previous observations

$153{ }^{24}$. Together, these results show that PulM is essential for PulA secretion under physiological 154 expression conditions and that PulG pilus assembly, although very inefficient, was not fully 155 abolished in the $\Delta p u l M$ mutant.

157 Effect of pul gene overexpression on piliation and secretion in the $\Delta \mathrm{pulM}$ mutants 
To further characterize the $\Delta p u l M$ mutant, we compared its piliation defect to that of

159 the previously characterized ApulI strain, which lacks PulI, one of the three minor

160 pseudopilins involved in assembly initiation ${ }^{35}$. As expected, the $\Delta p u l M$ mutant was more

161 deficient in pilus assembly compared to the ApulI strain (Figs. 2A and B). Analysis of the

162 same strains in liquid culture conditions showed that pul gene overexpression did not improve

163 PulA secretion in $\Delta p u l E, \Delta p u l G$ and $\Delta p u l I$ mutants. However, more than $10 \%$ of PulA was

164 secreted in the $\Delta p u l M$ mutant (Fig. 2C and 2D), confirming previous findings ${ }^{24}$ and

165 suggesting that overproduction of another Pul component might compensate for the absence 166 of PulM.

167 To test whether PulG overproduction allows to overcome this defect, we transformed

168 the pul mutant strains used above with plasmid pCHAP8568 (Table 1) carrying pulG under 169 the control of the lacZ promoter. While increased PulG levels did not overcome the

170 requirement for the PulE ATPase, piliation was improved in $\Delta p u l I+p u l G$ to an extent

171 comparable to that observed for the positive control $(\Delta p u l G+p u l G)$ (Fig. $2 \mathrm{E}$ and F). In

172 contrast, PulG overproduction led only to a marginal increase of PulG in the sheared fraction

173 of the $\Delta p u l M$ mutant, suggesting that PulM is nearly essential, either for T2SS assembly and

174 integrity or for its activity.

176 Analysis of pulM phenotype by immunofluorescence (IF) microscopy

178 To assess in more detail the piliation defect of the $\Delta p u l M$ strain, we analysed samples 179 of plate-grown bacteria using IF microscopy and anti-PulG antibodies (Materials and 180 Methods). PulG fibres were detected in strain producing the complete Pul T2SS (WT) and not 181 in the negative controls lacking the PulE ATPase or PulG (Fig. 3A, $\Delta E$ and $\Delta G$ ). Consistent 182 with the results of the shearing assay, fewer extracellular fibres were detected in the absence 
183 of PulM (Fig. 3A, $\Delta M$ ) compared to the $\Delta p u l I$ mutant (Fig. 3A, $\Delta l$ ) characterised previously

$184{ }^{35}$. While WT strain produced on average $0.47 \pm 0.09$ fibres per bacterium, the $\Delta p u l I$ and $185 \Delta p u l M$ strains produced on average 4-fold and 52-fold fewer fibres per bacterium, 186 respectively (Fig. 3B). The measurements of the length (Y-axis) of individual fibres (dots)

187 show that, compared to WT strain, the $\Delta p u l I$ and $\Delta p u l M$ strains produced longer fibres on 188 average (Fig. 3B). Plotting the relative frequency distributions of the fibre length in WT and $189 \Delta p u l I$ and $\triangle p u l M$ mutants further illustrates this tendency (Fig. 3C).

190 The increased PulG levels led to an increase in fibre numbers and median length to a

191 similar extent in all strains (Fig. 4A, B and D). However, while PulG overproduction restored 192 piliation in $\Delta p u l G$ and $\Delta p u l I$ strains, in the $\Delta p u l M$ mutant strain, the piliation defect remained

193 dramatic with only $\sim 10 \%$ of PulG found in the sheared fraction (Fig. 2E).

195 Differential effect of PulG ${ }^{E 5 A}$ and $P u l G^{T 2 A}$ on pseudopilus assembly and PulA secretion

We showed recently that E5A substitution in PulG strongly affects PulM binding,

198 raising a possibility that functional defects of $\mathrm{PulG}^{\mathrm{E} 5 \mathrm{~A}}$ could be due to the effect on PulG-

199 PulM interaction ${ }^{25}$. Residue E5 is localized in the highly conserved N-terminal TMS of major 200 and minor pseudopilins ${ }^{33}$. Another highly conserved polar residue in this segment of mature 201 pseudopilins and T4 pilins is Thr at position 2 (T2) (Fig. 5A). Interestingly, T2 and E5 show a 202 degree of co-variation when different pseudopilin sequences are aligned (Fig. 5B). This is 203 linked to the absence of E5 in the GspK family of T2SS pseudopilins and to the conservation 204 of A2 residue in these proteins (Fig. S1). To further characterize this conserved N-terminal 205 region of $\mathrm{PulG}$, we generated the $\mathrm{PulG}^{\mathrm{T} 2 \mathrm{~A}}$ variant and tested its interaction with PulM using 206 the BAC2H approach ${ }^{36}$. Full-length PulM and mature PulG or its variants fused to the Cterminus of T18 and T25 fragments of the catalytic domain of the CyaA adenylyl cyclase 
208 from Bordetella pertussis were co-produced in an E. coli $\Delta c y a$ mutant strain DHT1 ${ }^{37}$. Fusion

209 protein interactions resulted in functional complementation and conversion of ATP into

210 cAMP, monitored using the $l a c Z$ gene as a reporter.

211 The high $\beta$-galactosidase activity of strains co-producing PulM- and PulG- hybrids

212 indicated their strong interaction (Fig. 5C), which was negatively affected by the E5A

213 substitution (Fig. 5C) ${ }^{25}$. Similarly, the T2A substitution in PulG led to reduced lacZ

214 expression, showing that T2 is also important for PulM-PulG interaction. All these PulG

215 variants were still able to form homo-dimers (Fig. 5C), which is in agreement with the

216 evidence that residues in the globular head, but not in the N-terminal TMS, drive PulG

217 dimerization ${ }^{34}$. As a control of specificity, we substituted another highly conserved residue of

218 PulG TMS, proline at position 22 (P22), by an alanine. The $\beta$-galactosidase activity of strains

219 co-producing PulM- and $\mathrm{PulG}^{\mathrm{P} 22 \mathrm{~A}}$ - chimera did not differ significantly from that of strains

220 producing the wild type PulM- and PulG- hybrids, showing a specific role of conserved

221 residues T2 and E5 in PulG interaction with PulM.

222 We next tested the impact of the T2A substitution on PulG function. A $\Delta p u l G$ strain

223 complemented with the pulG(T2A) allele showed a $\sim 50 \%$ reduced ability to assemble T2SS

224 pili (Fig. 5D and 5E). Likewise, secretion of PulA was reduced by $\sim 50 \%$ for the $\mathrm{PulG}^{\mathrm{T} 2 \mathrm{~A}}$

225 variant, when all of the pul genes were co-expressed at physiological levels (Fig. 5F and 5G),

226 showing a phenotype strikingly different from $p u l G(E 5 A)$ mutants.

227 Analysis by IF microscopy showed similar trends. In strain carrying $p u l G(T 2 A)$ allele,

228 two-fold fewer fibres were observed relative to $p u l G^{\mathrm{WT}}$, and the defect was less severe than

229 for $\Delta p u l M+p u l G$ and $\Delta p u l G+p u l G(E 5 A)$ (Fig. 4A). Surprisingly, surface fibres in $\Delta p u l G+$

$230 \mathrm{pulG}(T 2 A)$ strain were shorter on average than those of the positive control $\Delta p u l G+p u l G$

231 (Fig. 4A, C and E). Although small, this difference is highly statistically significant and raises 
232 the possibility that the T2A mutation might also affect fibre elongation, for example by

233 impairing recruitment of PulG subunits to the AP.

235 PulGinteraction with PulM in the context of the Pul T2SS

Despite their functional differences, in the BAC2H assay the $\mathrm{PulG}^{\mathrm{T} 2 \mathrm{~A}}$ and $\mathrm{PulG}^{\mathrm{E} 5 \mathrm{~A}}$

238 variants were similarly impaired in interaction with PulM. To test whether PulG and PulG ${ }^{\text {E5A }}$

239 interacted with PulM in the context of the complete T2SS, we used a cross-linking approach.

240 E. coli PAP7460 bacteria moderately overproducing the Pul T2SSs and different PulG

241 variants were treated with $0.6 \%$ formaldehyde (FA), and complexes containing PulM were

242 detected using anti-PulM antibodies (Materials and Methods). In addition to PulM monomers

243 and homo-dimers, we observed a $\sim 38 \mathrm{kDa}$ band that corresponds to a PulG-PulM heterodimer

244 (Fig. 6, G-M), as it was only present in strains producing PulG (Fig. 6). Further supporting its

245 identity as a G-M heterodimer, this band showed shifts in migration dependent on the

246 molecular mass of co-produced PulG and its variants containing a C-terminal hexahistidine

247 tag (Fig. 6, lanes 3, 4) or containing, in addition, the 6-residue long N-terminal pre-peptide (in

248 strain lacking PulO, Fig. 6, lane 2). The levels of PulM-PulG ${ }^{\mathrm{E} \mathrm{A}_{-}}-\mathrm{His}_{6}$ heterodimer were

249 reduced relative to PulM-PulG-His 6 . However, consistent with the PulM-PulG interaction

250 study ${ }^{25}$, the E5A substitution in PulG did not fully abolish its interaction with PulM in the

251 context of the T2SS, failing to fully account for its dramatic effect on PulG assembly and

252 function.

254 Molecular dynamics simulations of PulG and its variants in POPE model membrane 
257 higher levels, even in the absence of other T2SS components ${ }^{25}$. We therefore hypothesized

258 that PulG $^{\mathrm{E} 5 \mathrm{~A}}$ might be blocked in the membrane prior to entry into the assembly pathway. To

259 test this possibility, we used atomistic MD simulations of PulG in model membranes in silico.

260 This approach allowed us to study conformations and dynamics of PulG in its native

261 environment and to quantify its atomic contacts with the membrane and solvent. Following

262 cleavage by prepilin peptidase, $\mathrm{PulG}$ is $\mathrm{N}$-terminally methylated at the conserved Phe residue

263 (hereafter designated MeF1) ${ }^{10}$. Previous analyses using mass spectrometry showed that most

264 of $\mathrm{PulG}^{\mathrm{E} 5 \mathrm{~A}}$ was methylated whereas $\sim 30 \%$ of $\mathrm{PulG}^{\mathrm{E} 5 \mathrm{~A}}$ that co-assembled with $\mathrm{PulG}{ }^{\mathrm{WT}}$ into

265 mixed pili remained non-methylated ${ }^{25}$. To compare all these forms of PulG, we embedded

266 structural models of a methylated $\mathrm{MePulG}^{\mathrm{WT}}$, as well as those of methylated $\mathrm{MePulG}^{\mathrm{E} 5 \mathrm{~A}}$ and

267 non-methylated PulG $^{\mathrm{E} 5 \mathrm{~A}}$ monomers, into 1-palmitoyl-2-oleoyl-sn-glycero-3-

268 phosphoethanolamine (POPE) model membranes, and performed independent triplicate 200-

269350 ns MD simulations of each.

270 Initial and representative final snapshots of these simulations (Fig. 7) show that, in the

271 final snapshot, the protein TMS became more deeply buried and its head domain bent towards

272 the lipid membrane surface to varying degrees. The exception was one of the $\mathrm{PulG}^{\mathrm{E} 5 \mathrm{~A}}$

273 simulations, wherein the bottom of the globular domain nevertheless interacted extensively

274 with the lipid by the end of the simulation. The solvent-accessible surface area (SASA) of

275 residues $60-125$, the bulk of the globular domain, decreased on average between $\sim 0.2$ and

$276 \sim 3.1 \mathrm{~nm}^{2}$ over the course of the simulations. Overall the MD simulations did not reveal any

277 statistically significant variations in protein conformation between variants, and SASA

278 suggested that the globular domain does not maintain strong, consistent contacts with POPE.

279 As expected, the E5A variants did not cause severe protein destabilization or membrane

280 perturbation, supporting the notion that the role of E5 is localized to the N-terminus. 
Next, we compared the maintenance of the protein secondary structure during each

282 simulation. In all the systems, the N-terminal $\alpha$-helix, with the exception of the first 4

283 residues, was almost entirely conserved throughout the simulations, as were the two $\beta$-sheets

284 between residues $97-102$ and 110-114. Transient disruptions to the $\alpha$-helix were observed in 285 one $\mathrm{PulG}^{\mathrm{E} 5 \mathrm{~A}}$ simulation (with residues $30-34$, or solely residue 34 , losing their helical 286 structure) and in one $\mathrm{MePulG}^{\mathrm{WT}}$ simulation (where the helix disintegrated around either 287 residues $27-30$ or again around residue 34 ). However, helix integrity was fully restored by the 288 end of these simulations. On the other hand, we observed significant differences in the 289 secondary structure of the five N-terminal residues between wild type and E5A variants. In all $290 \mathrm{MePulG}^{\mathrm{WT}}$ simulations the first several residues demonstrated a relaxation of helical structure 291 into either turns or coils, as illustrated in Figure 8. In contrast, in the MePulG ${ }^{\mathrm{E} 5 \mathrm{~A}}$ and $\mathrm{PulG}^{\mathrm{E} 5 \mathrm{~A}}$ 292 systems, the five N-terminal residues maintained their helical structure throughout, except in 293 one Me-PulG ${ }^{\mathrm{E} 5 \mathrm{~A}}$ simulation, where six N-terminal residues transiently formed a 3-10 helix or

294 turn structure between $\sim 150-200$ ns. Together, these results suggested that the residue E5 295 affects the secondary structure of the PulG N-terminus.

296 To study this phenomenon more closely, we quantified the proximity of E5 to the N297 terminus in all simulations. The distance between the centres-of-mass (COM) of residues 1 298 and 5 was largely fixed due to the helix on which they are both located. However, significant 299 differences were observed in the minimum distance between the residue 1 methyl group or 300 amide terminus (depending on whether the system contained MeF1 or F1) and any atom of 301 the residue 5 side-chain (Fig. 9A). Notably, in the MePulG ${ }^{\mathrm{WT}}$ system the methyl group of $302 \mathrm{MeF} 1$ and the E5 side-chain remained consistently within $3 \AA$ of each other, indicating their 303 electrostatic contact. This was not the case in the simulations of PulG variants carrying the 304 E5A substitution. In the PulG ${ }^{\mathrm{E} 5 \mathrm{~A}}$ system, the A5 side-chain made no contact with $\mathrm{F} 1$ and 305 remained at $6 \AA$ distance, only approaching to within $5 \AA$ in two simulations, and solely in the 
306 first 30 or $70 \mathrm{~ns}$. The $\mathrm{MePulG}^{\mathrm{E} 5 \mathrm{~A}}$ simulations showed more fluctuation, with the atoms

307 approaching to within $\sim 2.5 \AA$ and moving as far as $10 \AA$ apart; however, the side-chains

308 mostly remained $\sim 6 \AA$ apart. Visual analysis shown in Fig. 9B supported these results; E5 and

$309 \mathrm{MeF} 1$ remained close in $\mathrm{MePulG}^{\mathrm{WT}}$, while in the $\mathrm{MePulG}^{\mathrm{E} 5 \mathrm{~A}}$ system A5 stayed embedded in

310 lipid whereas MeF1 was extensively solvated. Importantly, in MePulG ${ }^{\mathrm{WT}}$ the $\mathrm{N}$-terminal

311 amine was engaged in interaction with E5 and did not form polar contacts with POPE

312 phosphate groups, while in $\mathrm{PulG}^{\mathrm{E} 5 \mathrm{~A}}$ it was anchored to three different POPE molecules

313 towards the end of the simulation (Fig. 9B). The $\mathrm{MePulG}^{\mathrm{E} 5 \mathrm{~A}}$ showed an intermediate

314 behaviour, with reduced amine interactions with POPE, perhaps explaining the fluctuating

315 distances to A5 during MD simulations. These data suggest that in MePulG ${ }^{\mathrm{WT}}$ E5 promotes

316 intra-molecular interactions with the $\mathrm{N}$-terminal positive charge of MeF1, thus reducing the

317 polar contacts with phospholipid head-groups, and potentially priming the protein for

318 membrane extraction during pseudopilus assembly. In contrast, the N-termini of the mutant

319 variants interacted with membrane phospholipids, anchoring the protein more firmly in the 320 bilayer.

321 Relative to the mutant systems, the $\mathrm{MePulG}^{\mathrm{WT}}$ experienced extended periods with no

322 hydrogen bonds to solvent water, and its MeF1 formed 0.6 hydrogen bonds to water, on

323 average. The $\mathrm{MeF} 1$ from $\mathrm{MePulG}^{\mathrm{WT}}$ and $\mathrm{MePulG}^{\mathrm{E} 5 \mathrm{~A}}$ proteins formed on average $\sim 0.9$

324 hydrogen bonds with POPE, relative to the $\sim 1.9$ hydrogen bonds formed by F1 from non-

325 methylated $\mathrm{PulG}^{\mathrm{E} 5 \mathrm{~A}}$. When normalised relative to the number of possible hydrogen bonds

326 from each, this analysis demonstrated that F1 engaged in hydrogen bonding to phospholipid

$32740 \%$ more than MeF1. The methylation therefore decreased the number of hydrogen bonds to

328 phospholipid head-groups, which might reduce the energetic cost of transferring the charged

329 N-terminus across the IM. 
$\mathrm{T} 2$ residue dynamics were also explored. $\mathrm{T} 2$ in $\mathrm{MePulG}^{\mathrm{WT}}$ formed only one hydrogen

331 bond to E5 during a single simulation frame, and only sporadic hydrogen bonds were 332 observed between the T2 hydroxyl oxygen and the MeF1 benzene ring. Instead, T2 formed on

333 average $\sim 1.4$ hydrogen bonds with water and $\sim 0.9$ hydrogen bonds with surrounding POPE 334 molecules. Likewise, in the $\mathrm{MePulG}^{\mathrm{E} 5 \mathrm{~A}}$ variant, $\mathrm{T} 2$ formed on average $\sim 1.3$ and $\sim 0.8$

335 hydrogen bonds to water and to POPE respectively, and did not form any bonds with MeF1.

336 Altogether, the simulations suggested that in the wild type, E5 engages in interaction with

337 MeF1, leaving T2 available for interactions with solvent, membrane or other proteins, 338 possibly PulM. The variation of residue 5 did not have a significant effect on T2 dynamics.

\section{Discussion}

To elucidate the early steps of pseudopilus assembly, we focused in this study on the

343 interactions of the major pseudopilin PulG with the PulM AP component of the K. oxytoca

344 T2SS. The results showed that PulM is fully required for PulA secretion under physiological

345 conditions. On the other hand, overproduction of T2SS components resulted in dramatically

346 reduced (but not fully abolished) PulA secretion and PulG fibre assembly in the pulM

347 knockout. This is in agreement with results obtained in $P$. aeruginosa lacking its homologue

348 XcpZ, which also shows low levels of piliation ${ }^{38}$. Quantitative IF microscopy analysis

349 indicated that pulM mutants assemble at least 50-fold less pili compared to wild type. This

350 defect is probably underestimated, since only fields containing at least one pilus were taken

351 into account during IF quantification (Materials and Methods). Contrary to our predictions,

352 the fibre length was not reduced but rather increased in $\Delta p u l M$ mutants. 
355 assembly of the functional T2SS complex ${ }^{25}$. The recent insights into the Myxococcus xanthus

356 T4P machinery from cryo-electron tomography reveal a continuous, envelope-spanning

357 apparatus ${ }^{12}$. Given the structural similarities between T4P and T2SSs, it is likely that the T2S

358 nano-machine is organized in a similar manner, so that PulM, together with PulL, might

359 constitute the lower periplasmic electron-dense ring in the periphery of the complex. In $M$.

360 xanthus, this ring is absent in bacteria lacking PilO, which is a probable PulM equivalent

361 sharing the same topology ${ }^{12}$. Studies of T2SS GFP chimera using live fluorescence

362 microscopy support the localization of the $V$. cholerae PulM homologue EpsM in fluorescent

363 foci dependent on the GspD, GspC and GspL homologues ${ }^{39}$. Since PulD-mCherry chimera

364 form similar fluorescent foci in the absence of other Pul factors, the secretin assembly

365 probably initiates the assembly of the T2SS complex ${ }^{40}$. These and other studies suggest that

366 PulM might serve as a link bringing together the pseudopilin/PulF complex and the PulD/C/L

367 complex through its interactions with PulG/PulH on one hand and PulL on the other 19;22; 23;

$362^{24 ; 25}$. Therefore, the observed critical role of PulM in fibre assembly could be due to its

369 requirement for building a functional T2SS machine and its role in PulL stabilization,

370 documented in several T2SSs $19 ; 22 ; 23 ; 24$. The rare PulG fibres that form in $\Delta p u l M$ mutants

371 under overexpression conditions might stem from the few T2SS complexes that assemble

372 through a low-affinity interaction of PulG with PulL ${ }^{25}$. The accumulation of unassembled

373 PulG in the IM would drive elongation of pili within these rare T2SS complexes, thereby

374 accounting for the higher median length of fibres in $\Delta p u l M$ mutants. A similar effect was

375 observed previously for the mutants lacking minor pseudopilins of the tip complex, including

376 Pull, used here as a control ${ }^{35}$.

377 In addition to the PulM role in PulL stabilization, its interaction with PulG in the

378 absence of other Pul factors ${ }^{25}$ suggested an additional, direct role in pseudopilus assembly.

379 Here, using chemical cross-linking, we showed that PulM and PulG also interact in the 
presence of other T2SS components, and that the E5A substitution reduced interaction with

381 PulM. Both PulG and PulM are integral membrane proteins that share the $\mathrm{N}$-in C-out 382 topology. In addition to the PulG residue E5, we show here that residue $\mathrm{T} 2$ in this region is 383 equally required for PulM interaction in the BAC2H assay, possibly accounting for residual 384 PulM binding of the PulG ${ }^{\mathrm{E} 5 \mathrm{~A}}$ variant. This is also supported by MD simulation results, where 385 residue T2 remained sterically available for inter-molecular interactions in all variants. The 386 conformation of $\mathrm{MePulG}^{\mathrm{WT}}$ is compatible with PulM binding via $\mathrm{T} 2$ residue, which would 387 explain the effects of PulG T2A substitution on PulM interaction and its impact on the fibre 388 length. Furthermore, given that in MD simulations residue E5 of mature MePulG ${ }^{\mathrm{WT}}$ is 389 engaged in interactions with the N-terminal amine, T2 might be an important determinant of 390 this interaction during the membrane extraction step of PulG assembly. Since T2 formed

391 hydrogen bonds with the surrounding solvent and membrane in all simulations, PulM binding 392 might shield T2 and reduce these contacts to facilitate PulG membrane extraction, or its 393 transfer to another binding partner. The high conservation of T2 and E5 among T2SS 394 pseudopilins and T4 pilins (Fig. 5A) further shows the importance of PulG-PulM interface, 395 whose disruption, at least on the PulG side, correlates with severe functional defects. Testing 396 this model requires mutagenesis studies of the PulM cytoplasmic tail and/or TMS. Although 397 no structural information on this part of PulM and its homologues is available, the predicted 398 topology of these regions makes them obvious candidates for PulG binding. Although the interaction with PulM was affected to a similar extent for PulG $^{\mathrm{E} 5 \mathrm{~A}}$ and $400 \mathrm{PulG}^{\mathrm{T} 2 \mathrm{~A}}$ variants in the BAC2H assay, the functional impact of the two substitutions was very 401 different. Compared to the E5A substitution that fully abolished function, the T2A 402 substitution resulted in a partial defect in both fibre assembly and protein secretion (Fig. 5). 403 The weaker PulM - PulG interaction reduced by half the number of assembled pili, which 404 might directly reflect the number of assembled T2SS complexes. In addition, the pseudopili 
405 composed of $\mathrm{PulG}^{\mathrm{T} 2 \mathrm{~A}}$ variant showed lower median length compared to wild type. This might

406 reflect reduced fibre stability, in agreement with recent structural analysis of meningococcal

407 T4P that have implicated residue T2 in inter-protomer interactions, involving residue E5 of

408 the protomer below ${ }^{41}$. However, the defective PulA secretion in the presence of PulG $^{\mathrm{T} 2 \mathrm{~A}}$

409 supports an early role of $\mathrm{T} 2$ in PulG assembly, consistent with previous studies showing that

410 PulG pilus stability is not required for secretion ${ }^{33 ; 34}$. In this model, PulM binding might

411 facilitate PulG recruitment to the machinery, e.g. by targeting PulG subunits to the active site

412 of the AP. In addition, PulM might act as a chaperone, favouring the PulG conformation

413 compatible with assembly. In $V$. cholerae, a cross-linking study showed direct interaction

414 between the major pseudopilin EpsG and the PulL homologue EpsL ${ }^{18}$, and our recent study

415 indicates weak interactions between PulL and $\mathrm{PulG}{ }^{25}$. While the absence of prepilin

416 peptidase in $V$. cholerae prevents the EpsG-EpsL cross-linking, in our studies the presence of

417 the prepeptide seems to enhance PulG-PulM interaction, as suggested by the similar levels of

418 heterodimers in the strain lacking PulO, which contained lower levels of PulG monomer (Fig.

419 6, anti-G, lane 2). The preferential binding of precursor and mature forms of major

420 pseudopilin to different partners in the AP - L or M - might reflect different conformations

421 that represent intermediates in the assembly pathway.

422 To gain insight into these PulG conformations in wild type and E5A mutant variants

423 we turned to MD simulations. Unexpectedly, this approach highlighted a role of another

424 interacting partner of PulG - the plasma membrane, which poses a major obstacle to assembly

425 of type 4 filaments in bacteria. By revealing the striking conformational difference between

426 the N-termini of $\mathrm{MePulG}^{\mathrm{WT}}$ compared to $\mathrm{MePulG}^{\mathrm{E} 5 \mathrm{~A}}$ and $\mathrm{PulG}^{\mathrm{E} 5 \mathrm{~A}}$ variants in the membrane,

427 the results of MD simulations provide a plausible explanation for the phenotypes of

428 pulG(E5A) mutants. Contrary to the $\mathrm{PulG}^{\mathrm{E} 5 \mathrm{~A}}$ whose N-terminal amine is firmly anchored in

429 the membrane through polar contacts with up to three different phospholipids, in $\mathrm{MePulG}^{\mathrm{WT}}$ 
430 residue E5 neutralized the N-terminal membrane anchor. The T4P assembly models proposed

431 by Craig et al. ${ }^{42}$ and Melville and Craig ${ }^{5}$ emphasise the importance of charge neutralisation

432 during fibre assembly. While these models consider the inter-protomer charge neutralisation,

433 the results of MD simulations and $(\mathrm{Me}) \mathrm{PulG}^{\mathrm{E} 5 \mathrm{~A}}$ membrane accumulation strongly support the

434 role of intra-molecular neutralisation of the N-terminal amine via a loop formation with E5.

435 The intra-molecular contacts between F1 and E5 residues have also been observed in the

436 crystal structure of T4 pilins of the P. aeruginosa PAK strain PilA (Protein database (PDB)

437 entry code: $1 \mathrm{OQW}$ ) and in $N$. gonorrhoeae pilin PilE (PDB: 2HI2) ${ }^{42 ;} 43$. A similar loop

438 structure is suggested by the MD analyses of the $P$. aeruginosa major T4P subunit PilA ${ }^{44}$.

439 Interestingly, in that study the loop is observed in initial stages of MD simulation, whereas

440 towards the end, when PilA is fully embedded in the membrane, the N-terminal amine

441 interacts with the phospholipid head-groups. The difference compared to our study is that

442 PilA was not modelled with a methyl group, which might have led to ceasing of the intra-

443 molecular F1-E5 interaction by the end of the simulation. Following the positive inside rule

$444^{45}$, the positively charged residues of IM proteins are anchored to the cytoplasmic face of the

445 IM through interactions with negatively charged phosphate groups of membrane

446 phospholipids. Besides, since the MD simulation conditions do not take into account the

447 proton gradient, the membrane anchoring of $\mathrm{MePulG}^{\mathrm{WT}}$ and $(\mathrm{Me}) \mathrm{PulG}^{\mathrm{E} 5 \mathrm{~A}}$ might be even

448 stronger in vivo and might represent a rate-limiting step during pseudopilus assembly. Strong

449 membrane association and stable N-terminal helix conformation could explain the decreased

450 turnover rate of the $\mathrm{PulG}^{\mathrm{E} 5 \mathrm{~A}}$ compared to $\mathrm{PulG}^{\mathrm{WT}}$ as measured previously ${ }^{34}$ and its relative

451 accumulation in $E$. coli $^{25}$.

452 The MD simulation results suggest that N-methylation might further reduce polar

453 contacts of pilins with the solvent, contributing to their extraction from the membrane. Our

454 observations using this approach also provide a clue for the link between E5 and N- 
455 methylation, observed previously. While the E5K or E5V substitutions in the major subunit of

456 P. aeruginosa T4P PilA abolish N-methylation 9; 46, the E5A substitution in PulG only

457 reduces the methylation efficiency by around $30 \%{ }^{25}$. Enhanced N-terminal amine contacts

458 with phospholipid head-groups in these variants might block or reduce the access of N-methyl

459 transferase domain of prepilin peptidase to its amine substrate. The different effects of E5

460 residue substitutions on T4 pilins and T2SS pseudopilins might be linked to the differences

461 between their AP components such as PulM, a hypothesis that requires further investigation.

463 and archaeal prepilin peptidases do not have a methyl-transferase domain and activity. One

464 possible explanation for these differences might lie in the different composition of archaeal

465 membranes, formed by tetraether lipids that are structurally and functionally different from

466 bacterial phospholipids ${ }^{48}$. Rare exceptions to this rule include Aeropyrum pernix and

467 Archaeoglobus fulgidus, where flagellins do have a conserved E5 residue, and whose

468 presence correlates with the documented presence of phospholipids in their membranes ${ }^{49 ; 50}$.

469 However, in most archaea the rules of membrane anchoring for proteins might be very

470 different compared to bacteria. The conserved E5 and N-methylation might represent a

471 bacterial solution to the problem of membrane escape for proteins anchored in the

472 phospholipid membrane. The remarkable efficiency of this strategy and the dramatic effect of

473 E5A mutation provide another example of the importance and the strength of weak bonds in

474 biological systems.

475

Overall, the results of this study are compatible with a dual role of PulM binding to

477 PulG. During initial steps of T2SS assembly, PulM might bind the PulG precursor to favour

478 recruitment of pseudopilin-PulF subcomplex to the secretin/AP complex ${ }^{25}$. During fibre

479 elongation, PulM might facilitate PulG targeting to the active site of the T2SS and/or its 
480 extraction from the membrane, by favouring a conformation that minimises membrane 481 contacts. These roles for PulM are based on its ability to bind PulG and PulH on one hand and 482 PulL on the other, probably in a dynamic fashion, as shown for its homologues in T2SSs ${ }^{51}$ 483 and T4P ${ }^{52}$. Eventually, PulG recruited from the IM pool would reach the assembly focus 484 defined by PulF and the PulE ATPase poised for membrane extraction and incorporation into 485 the pseudopilus. Future studies are needed to reveal the nature of these conformational 486 changes and the precise molecular function of PulM in coordinating PulG membrane 487 extraction with its incorporation into the growing fibre.

\section{Materials and Methods}

\section{Bacterial strains and culture}

The E. coli DH5 $\alpha$ F' lacI $I^{Q}$ strain was used for cloning purposes. Strain PAP7460

$494\left[\Delta\left(\right.\right.$ lac-argF)U169 araD139 relA1 rpsL150 $\Delta$ malE444 malG501 [F' (lacI ${ }^{Q}$ DlacZM15 pro+ $495 \operatorname{Tn} 10)]\left(T c^{R}\right)^{24}$ was used for $p u l$ gene expression and strain PAP5299 [araD139 $\Delta(\arg F$ 496 lac)U169 rpsL150 relA1 flb5301 deoC1ptsF25 thi pcnB::Tn10 (F'lacl $\left.{ }^{Q}\right){ }^{33}$ was employed 497 for secretion assays. Bacteria were grown at $30{ }^{\circ} \mathrm{C}$ in $\mathrm{LB}$ medium $\left(10 \mathrm{~g} \mathrm{~L}^{-1}\right.$ bacto tryptone; $5 \mathrm{~g}$ $498 \mathrm{~L}^{-1}$ yeast extract; $10 \mathrm{~g} \mathrm{~L}^{-1} \mathrm{NaCl}$; $\mathrm{pH}$ 7.0) containing antibiotics as required: ampicillin, $100 \mu \mathrm{g}$ $499 \mathrm{ml}^{-1}$; chloramphenicol, $25 \mu \mathrm{g} \mathrm{ml}^{-1}$; kanamycin, $20 \mu \mathrm{g} \mathrm{ml}^{-1}$. Expression of pul genes cloned 500 under the control of the lac promoter was induced with $1 \mathrm{mM}$ isopropyl- $\beta$-D-thiogalactoside 501 (IPTG). Expression of pul genes was induced with $0.4 \%$ maltose in LB medium buffered with $5021 / 10$ volume of M63B1 salt solution (modified from ${ }^{53}$ : $13.6 \mathrm{~g} \mathrm{~L}^{-1} \mathrm{KH}_{2} \mathrm{PO}_{4} ; 2 \mathrm{~g} \mathrm{~L}^{-1}$ $503\left(\mathrm{NH}_{4}\right)_{2} \mathrm{SO}_{4} ; 0.2 \mathrm{~g} \mathrm{~L}^{-1} \mathrm{MgSO}_{4} \cdot 7 \mathrm{H}_{2} \mathrm{O} ; 0.5 \mathrm{mg} \mathrm{L}^{-1} \mathrm{FeSO}_{4} \cdot 7 \mathrm{H}_{2} \mathrm{O} ; 1 \mathrm{mg} \mathrm{L}{ }^{-1}$ vitamin $\left.\mathrm{B} 1 ; \mathrm{pH} 7.0\right)$. 
505 Recombinant DNA and plasmid construction

506 The list of plasmids used in this study is shown in Table 1. 
507 Table 1. Plasmids used in this study.

508

\begin{tabular}{|c|c|c|c|}
\hline Name & Ori / Resistance ${ }^{a}$ & \begin{tabular}{|l} 
Relevant \\
characteristics
\end{tabular} & Reference \\
\hline pCHAP8185 & ColE1 / Ap ${ }^{R}$ & pul $A_{\text {sol }}$ all pul genes & 35 \\
\hline pCHAP8184 & ColE1 / Ap ${ }^{R}$ & $\begin{array}{l}p u l A_{\text {sol }} \text { all pul genes } \\
\Delta p u l G\end{array}$ & 33 \\
\hline pCHAP8400 & ColE1 / Ap ${ }^{R}$ & $\begin{array}{l}\text { All pul genes } \Delta p u l G \\
\Delta p u l N O\end{array}$ & This study \\
\hline pCHAP8200 & ColE1 / Ap ${ }^{R}$ & $\begin{array}{l}\text { pulA } A_{\text {sol }} \text { all pul genes } \\
\Delta \text { pulE }\end{array}$ & This study \\
\hline pCHAP8218 & ColE1 / $A p^{R}$ & $\begin{array}{l}\text { pulA } A_{\text {sol }} \text { all pul genes } \\
\Delta \text { pull }\end{array}$ & 35 \\
\hline pCHAP1217 & ColE1 / Ap ${ }^{R}$ & All pul genes $\Delta p u l L$ & 24 \\
\hline pCHAP8496 & ColE1 / Ap ${ }^{R}$ & $\begin{array}{l}\text { pulA } A_{s o l} \text { all pul genes } \\
\Delta p u l M\end{array}$ & This study \\
\hline pCHAP8251 & ColE1 / Ap ${ }^{R}$ & $\begin{array}{l}\text { pulA } A_{s o l} \text { all pul genes } \\
\Delta p u l L\end{array}$ & This study \\
\hline pCHAP8811 & ColE1 / Ap ${ }^{R}$ & pulM & \begin{tabular}{|l|} 
This study \\
\end{tabular} \\
\hline pCHAP576 & ColE1 / Kan ${ }^{R}$ & pulO & 54 \\
\hline pCHAP8258 & $\mathrm{p} 15 \mathrm{~A} / \mathrm{Cm}^{\mathrm{R}}$ & pulL & \begin{tabular}{|l|} 
This study \\
\end{tabular} \\
\hline pCHAP1353 & $\mathrm{p} 15 \mathrm{~A} / \mathrm{Cm}^{\mathrm{R}}$ & pulM & \begin{tabular}{|l|}
24 \\
\end{tabular} \\
\hline pCHAP8843 & $\mathrm{p} 15 \mathrm{~A} / \mathrm{Cm}^{\mathrm{R}}$ & pulL-pulM & This study \\
\hline pCHAP1362 & $\mathrm{p} 15 \mathrm{~A} / \mathrm{Cm}^{\mathrm{R}}$ & pulG-his $_{6}$ & \\
\hline pCHAP7785 & $\mathrm{p} 15 \mathrm{~A} / \mathrm{Cm}^{\mathrm{R}}$ & pulG $^{E 5 A}-$ his $_{6}$ & This study \\
\hline pCHAP8658 & $\mathrm{p} 15 \mathrm{~A} / \mathrm{Cm}^{\mathrm{R}}$ & pulG & \begin{tabular}{|l|}
34 \\
\end{tabular} \\
\hline pCHAP8732 & $\mathrm{p} 15 \mathrm{~A} / \mathrm{Cm}^{\mathrm{R}}$ & $p u l G^{T 2 A}$ & This study \\
\hline pCHAP8663 & $\mathrm{p} 15 \mathrm{~A} / \mathrm{Cm}^{\mathrm{R}}$ & $p{ }^{E} G^{E 5 A}$ & \begin{tabular}{|l|}
34 \\
\end{tabular} \\
\hline pCHAP8875 & ColE1 / Ap ${ }^{R}$ & $\begin{array}{l}p_{\text {ul }} A_{\text {sol }} \text { all pul genes } \\
\Delta p u l G \Delta p u l M\end{array}$ & This study \\
\hline pCHAP8377 & ColE1 / Ap ${ }^{R}$ & pulKLMNO & This study \\
\hline pCHAP8395 & ColE1 / Ap ${ }^{R}$ & pulKLM & This study \\
\hline pSU19 & $\mathrm{p} 15 \mathrm{~A} / \mathrm{Cm}^{\mathrm{R}}$ & Empty vector & 56 \\
\hline pBGS18 & ColE1 $/ \mathrm{Km}^{\mathrm{R}}$ & Empty vector & 57 \\
\hline pUT18c & ColE1 / Ap ${ }^{R}$ & placZ-T18 & 36 \\
\hline pKT25 & $\mathrm{p} 15 \mathrm{~A} / \mathrm{Km}^{\mathrm{R}}$ & placZ-T25 & 36 \\
\hline pCHAP8154 & ColE1/Ap ${ }^{R}$ & pUT18c pulM & 25 \\
\hline pCHAP8155 & $\mathrm{p} 15 \mathrm{~A} / \mathrm{Km}^{\mathrm{R}}$ & pKT25 pulM & 25 \\
\hline pCHAP7330 & ColE1/Ap ${ }^{R}$ & pUT18c pulG & \begin{tabular}{|l|l|}
34 \\
\end{tabular} \\
\hline pCHAP7332 & $\mathrm{p} 15 \mathrm{~A} / \mathrm{Km}^{\mathrm{R}}$ & pKT25 pulG & 34 \\
\hline pCHAP8670 & ColE1/Ap ${ }^{R}$ & pUT18c pulG (E5A) & 34 \\
\hline pCHAP8420 & $\mathrm{p} 15 \mathrm{~A} / \mathrm{Km}^{\mathrm{R}}$ & pKT25 pulG (E5A) & 34 \\
\hline pCHAP8733 & ColE1/Ap ${ }^{R}$ & pUT18c pulG (T2A) & This study \\
\hline pCHAP8734 & $\mathrm{p} 15 \mathrm{~A} / \mathrm{Km}^{\mathrm{R}}$ & pKT25 pulG (T2A) & This study \\
\hline pCHAP8429 & ColE1/Ap ${ }^{R}$ & pUT18c pulG (P22A) & This study \\
\hline pCHAP8482 & $\mathrm{p} 15 \mathrm{~A} / \mathrm{Km}^{\mathrm{R}}$ & pKT25 pulG (P22A) & This study \\
\hline
\end{tabular}

509 a. Ap, ampicillin; $\mathrm{Cm}$, chloramphenicol; $\mathrm{Km}$, kanamycin.

510 Plasmid pCHAP8200 was generated by replacing the 7430-bp EcoRI-Bsu36I fragment

511 of plasmid pCHAP8185 with the corresponding fragment of plasmid pCHAP1230 carrying 
512 the $\Delta p u l E$ allele ${ }^{24}$. Plasmid pCHAP8251 was generated by replacing the EcoRI-HindIII

513 fragment of plasmid pCHAP8185 with the corresponding fragment from pCHAP1217

514 plasmid carrying the nonpolar deletion of the pulL gene. Plasmid pCHAP8496 carrying a

515 complete in-frame deletion of pulM was generated as follows. Random insertions of the GPS5

$516\left(\mathrm{Km}^{\mathrm{R}}\right)$ cassette in the pulM gene were generated in vitro using the GPS ${ }^{\circledR}$-LS kit (New England

517 Biolabs). Two insertions of the GPS5 cassette, each marked with a single PmeI site mapping

518 in positions 20 and 120 of pulM ORF, were combined in plasmid pCHAP1353 to give

519 pCHAP8370. The pulM::kan allele was introduced into pUC18 derivative containing the

520 pulM gene, pCHAP8510, to give pCHAP8512. This plasmid was digested with PmeI to create

521 an in-frame deletion of pulM, giving pCHAP8513. The DraIII-NsiI fragment containing the

$522 \Delta p u l M$ allele was ligated to the DraIII-NsiI fragment of pCHAP8377 containing the distal half

523 of the pul operon, which was obtained by self ligating the AleI fragment of pCHAP231. This

524 yielded plasmid pCHAP8516. In the final step, the NotI-EcoRI A fragment of pCHAP8516

525 was ligated with the large EcoRI-NotI fragment of plasmid pCHAP8185 to give pCHAP8496.

526 Plasmid pCHAP8811 was constructed by sub-cloning the EcoRI-HindIII insert containing the

527 pulM gene from plasmid pCHAP1353 into pUC18 vector digested with EcoRI and HindIII.

528 Plasmid pCHAP8732 carrying $p u l G(T 2 A)$ allele was generated by Quick-change mutagenesis

529 using primers pulGT2A-5 and pulGT2A-3 (Table S1). Primers PulG T2A-Kpn and PulG Eco-

5303 were used to amplify the pulG(T2A) allele from plasmid pCHAP8732 in KpnI and EcoRI

531 digested BAC2H plasmids pUT18c and pKT25, to yield pCHAP8733 (pUT18c-pulG ${ }^{\mathrm{T} 2 \mathrm{~A}}$ ) and

532 pCHAP8734 (pKT25-pulG ${ }^{\mathrm{T} 2 \mathrm{~A}}$ ). Plasmid pCHAP8258 was made by cloning the pulL gene

533 PCR-amplified with primers PulL-Eco 5 and PulL-Hind 3 in the EcoRI and HindIII sites of

534 pSU18. Plasmid pCHAP8843 was generated by cloning the PCR amplified pCHAP8185

535 fragment containing the pulL and pulM genes in pSU18 EcoRI and HindIII sites amplified by

536 PCR using PulL-Eco 5 and PulM 3 oligonucleotide primers. All PCR reactions were 
537 performed using the high-fidelity Pwo polymerase (Roche). Plasmid pCHAP8732 was

538 derived from pCHAP8658 by the Quick-change method of site-directed mutagenesis with 539 primers PulG T2A-5 and PulG T2A-3 (Table S1). Plasmid pCHAP8875 was constructed by

540 ligating the pCHAP8184 NotI-HindIII B fragment with the pCHAP8496 NotI-HindIII

541 fragment A. Plasmid pCHAP8400 was made in several steps. First, plasmid pCHAP8377 was

542 constructed by ligating the AleI fragment of pCHAP231 containing the distal half of the pul

543 operon. The EcoNI fragment of pCHAP8377 containing pulN and pulO genes was deleted to

544 give pCHAP8395. The EcoRI-NotI fragments from pCHAP8395 and pCHAP8184 were then

545 combined to reconstitute the pul operons with deletions of $p u l G, p u l N$ and pulO.

546 All plasmid constructs were verified by sequencing (GATC). The list of 547 oligonucleotides is provided in Table S1.

549 In vivo cross-linking and PulM-PulG interaction analysis.

For the formaldehyde cross-linking, $40 \mathrm{OD}_{600}$ of bacterial cultures were centrifuged

552 (3500 x g, 5 minutes, room temperature). Bacterial pellets were washed with $10 \mathrm{ml}$ PBS and

553 resuspended in PBS at $8 \mathrm{OD}_{600} \mathrm{ml}^{-1}$. Following the cross-linking with $0.6 \%$ formaldehyde

554 (Sigma-Aldrich) for 20 minutes at $30^{\circ} \mathrm{C}$ with vigorous shaking, the bacteria were pelleted for

5552 minutes at $\sim 12000 \mathrm{xg}$ and resuspended in $5 \mathrm{ml}$ of $50 \mathrm{mM}$ Tris- $\mathrm{HCl}(\mathrm{pH} 8.0)$ for 10 minutes

556 at room temperature. The quenched reactions were centrifuged at $8000 \mathrm{xg}$ for 5 minutes and

557 the pellets were resuspended in $2 \mathrm{ml}$ of cold TBS (20 mM Tris- $\mathrm{HCl}, 150 \mathrm{mM} \mathrm{NaCl}, \mathrm{pH} 7.4)$

558 containing cOmplete ${ }^{\mathrm{TM}}$ ULTRA EDTA-free (Roche) protease inhibitor cocktail

559 (concentration as indicated by the supplier). The cells incubated on ice for 10 minutes in the

560 presence of $0.2 \mathrm{mg} \mathrm{ml}^{-1}$ lysozyme and $0.02 \mathrm{mg} \mathrm{ml}^{-1}$ DNase I, followed by sonication at $4^{\circ} \mathrm{C}$

561 using the Vibra-Cell Ultrasonic Processor 75186 (Sonics \& Materials) at 30\% amplitude with 
$5621 \mathrm{sec}$ ON / $1 \mathrm{sec}$ OFF cycles for a total sonication time of 10 seconds. The total cell extracts

563 were analysed by SDS-PAGE and immunodetection as described below.

565 Piliation and secretion assays

$567 \quad$ Functional assays to test piliation and secretion were performed as described in ${ }^{34}$. For

568 the shearing assay, bacteria were grown overnight in LB agar plates and expression of pul

569 genes was induced by maltose (and IPTG, when required). Bacteria were scraped off the

570 plates and resuspended in $\mathrm{LB}$ at $1 \mathrm{OD}_{600} \mathrm{ml}^{-1}$, and $1 \mathrm{ml}$ of the suspensions was vortexed for 1

571 minute to detach surface pili. Upon centrifugation of the samples at $\sim 12000 \mathrm{x} g$ for 5 minutes

572 at $4^{\circ} \mathrm{C}$, the pelleted bacteria were resuspended in SDS sample buffer, and the supernatants

573 containing the sheared pili were submitted to TCA (trichloroacetic acid) precipitation. Briefly,

574 the supernatants were centrifuged at $\sim 12000 \mathrm{x}$ g for another 10 minutes at $4^{\circ} \mathrm{C}$ to remove any

575 remaining bacteria, and pili were precipitated in $10 \%$ trichloroacetic acid for 30 minutes on

576 ice. The precipitates were pelleted by centrifugation at $\sim 12000 \mathrm{x}$ g for 30 minutes at $4^{\circ} \mathrm{C}$,

577 washed twice with cold $\left(-20^{\circ} \mathrm{C}\right)$ acetone, air-dried and resuspended in SDS sample buffer.

578 Equal amounts of cell and sheared fractions were analysed using SDS-PAGE and PulG

579 immunodetection. For the secretion assay, bacteria producing a non-acylated, soluble variant

580 of PulA were grown in LB medium buffered with 0.1 volume of M63 salts, supplemented

581 with $1 \mathrm{mM}$ IPTG and $0.2 \%$ maltose for the induction of pul genes. After normalization of 582 cultures to $2 \mathrm{OD}_{600} \mathrm{ml}^{-1}, 1 \mathrm{ml}$ was centrifuged at $\sim 12000 \mathrm{x} \mathrm{g}$ for 5 minutes at $4^{\circ} \mathrm{C}$. The

583 pelleted cells were resuspended in SDS sample buffer. The supernatants were centrifuged

584 again under the same conditions, and a sample was taken from the topmost part of the tube

585 and mixed with 2 x SDS sample buffer. The same OD equivalent amounts of cell and

586 supernatant fractions were analysed by SDS-PAGE and immunodetection of PulA. 
Protein separation was performed by SDS-PAGE in Tris-tricine gels ${ }^{58}$ containing

$59110 \%$ acrylamide, using Appelex or BioRad vertical gel electrophoresis systems. Proteins were

592 electro-transferred onto Amersham Hybond ECL nitrocellulose membranes (GE Healthcare)

593 using the semi-dry method with a buffer containing $5.8 \mathrm{~g} \mathrm{~L}^{-1}$ Tris base, $2.9 \mathrm{~g} \mathrm{~L}^{-1}$ glycine, $20 \%$

594 ethanol, $0.026 \%$ SDS. Membranes were blocked with 5\% skim milk in TBST (10mM Tris$595 \mathrm{HCl}, 15 \mathrm{mM} \mathrm{NaCl}, 0.05 \%$ Tween20, $\mathrm{pH}$ 7.5-7.6), probed for $1 \mathrm{~h}$ with specific antiserum 596 (1:2000 anti-PulG, 1:2000 anti-PulA, 1:1000 anti-PulL, 1:500 anti-PulM, 1:2000 anti-LamB, 597 1:10000 anti-RbsB), washed several times with TBST, incubated with secondary antibody 598 (1:20000 or 1:40000 anti-rabbit, horseradish peroxidase-coupled) for $1 \mathrm{~h}$, and washed 599 extensively with TBST. Membranes were developed by enhanced chemiluminescence using 600 Pierce ECL 2 (Thermo Scientific), Western Lightning Plus ECL (PerkinElmer) or 601 SuperSignal West Femto (Thermo Scientific), and the signal was recorded using Typhoon 602 FLA 9000 imager (GE Healthcare) or LAS 4000 imager (Fujifilm). ImageJ software ${ }^{59}$ was 603 used for densitometric analysis of bands.

604

605 Bacterial two-hybrid assay

607 Competent cells of strain DHT1 ${ }^{37}$ were co-transformed with pUT18C and pKT25 608 derivatives and bacteria were grown for $48 \mathrm{~h}$ at $30^{\circ} \mathrm{C}$ on LB plates containing Ap and $\mathrm{Km}$. Six 609 colonies were picked at random and inoculated into $5 \mathrm{ml}$ cultures in LB containing $\mathrm{Km}$ and 610 Ap, grown overnight and inoculated the next day into fresh medium containing $1 \mathrm{mM}$ IPTG. 611 Bacteria were cultured to mid-log phase and $\beta$-galactosidase activity was measured as 
612 described ${ }^{60}$. At least 2 independent experiments were performed with several randomly

613 picked transformants. Bar graphs represent mean values and error bars indicate standard

614 deviation. The non-parametric Kruskal-Wallis followed by Dunn's multiple comparison tests

615 were used in statistical analysis using the Graphpad Prism 6 software.

617 Immunofluorescence microscopy

IF labelling of pili was performed as described previously ${ }^{34}$. Bacteria grown for 16

620 hours at $30^{\circ} \mathrm{C}$ on LB agar supplemented with $0.4 \%$ maltose were carefully resuspended in

621 PBS at $1 \mathrm{OD}_{600} \mathrm{ml}^{-1}$ and immobilized on coverslips coated with poly-L-lysine. After 30

622 minutes of fixation using 3.7\% formaldehyde at room temperature, reactions were quenched

623 with $1 \mathrm{M}$ Tris- $\mathrm{HCl} \mathrm{pH} 8.0$ and samples were blocked with $1 \%$ bovine serum albumin (BSA) in

624 PBS. PulG surface pili were detected using an anti-PulG antibody (1:1000) and a secondary

625 anti-rabbit IgG coupled to Alexa Fluor 488 (1:200); bacteria were stained with 4',6-

626 diamidino-2-phenylindole (DAPI). Samples were observed with an inverted Leica DMRIBe

627 fluorescence microscope, and images were acquired with a Cool-Snap HQ CCD camera

628 (Roper's Instruments). Pili number and length were quantified semi-automatically using the

629 Metamorph software 6.1 (Universal Imaging) after having applied a shape filter to the images

630 to omit round-shaped unspecific dots, which were present in all samples including the

631 negative controls; the bacteria were counted manually using the same software. The non-

632 specific labelling was not taken into consideration for the quantification. Note that since

$633>95 \%$ of the fields in the $\Delta p u l M$ and $\Delta p u l M+p u l G$ mutants did not have any pili, we defined

634 a relevant field of vision as a field containing at least one pilus, and only relevant fields were

635 taken into account for the quantification. The statistical significance of the differences was

636 assessed by a Kruskal-Wallis test followed by a post-hoc analysis using the Dunn's multiple 
637 comparison tests. Graphs corresponding to pili number and length generated were built using

638 KaleidaGraph 4.1.

640 Molecular dynamics simulations

A full-length model of PulG was derived from the X-ray crystal structure of the $K$.

643 oxytoca PulG periplasmic domain (PDB ID: 1T92), as described in ${ }^{33}$. The missing 20

644 carboxy-terminal residues were modelled on the basis of close homology to GspG from

645 EHEC, and the TMS was modelled from PilA of Pseudomonas aeruginosa ${ }^{33}$. All residue

646 mutations were performed using PyMOL (The PyMOL Molecular Graphics System, Version

647 1.8 Schrödinger, LLC). The final $\mathrm{PulG}^{\mathrm{WT}}$ structure was composed of 133 amino acids and

648 included one calcium cation; all ionisable groups were assigned to their most probable

649 charged states at neutral $\mathrm{pH}$.

650 PulG variants were then embedded in a pre-equilibrated palmitoyl-oleoyl

651 phosphatidylethanolamine (POPE) membrane, using the GROMACS g_membed tool ${ }^{61}$. PulG

652 position along the z-axis was guided by the presence of non-polar and hydrophobic residues

653 in the lower section of the $\alpha$-helix, and interfacial aromatic residue Trp94. The systems were

654 solvated with TIP3P water, via superimposition of a pre-equilibrated box of waters, and a

$655 \sim 0.1 \mathrm{M}$ concentration of $\mathrm{NaCl}$, with dimensions $\sim 90 \times 90 \times 162 \AA$. The resulting systems

656 contained $\sim 36,500$ water molecules and 314 lipids.

657 At each stage of system setup, steepest descent energy minimization was performed to 658 relax the protein geometry and to remove steric clashes between protein/lipid/solvent. The 659 system was equilibrated over $1.5 \mathrm{ns,} \mathrm{during} \mathrm{which} \mathrm{position} \mathrm{restraints,} \mathrm{applied} \mathrm{to} \mathrm{all} \mathrm{non-}$ 660 hydrogen protein atoms, were gradually removed to relax the protein structure, membrane and 661 solvent. The $\mathrm{Ca}^{2+}$ ion was unrestrained during the equilibration steps yet remained in the 
662 original bound position. Finally, 200-350 ns production MD simulations were carried out. All

663 simulations were performed using GROMACS ${ }^{62}$ version $4.5^{63}$. The protein was treated using

664 the CHARMM22/CMAP force field ${ }^{64}$, and POPE lipid molecules using the CHARMM36

665 parameter set ${ }^{64}$. The parameters for MeF1 were formulated based on existing parameterized

666 fragments. Equations of motion were integrated using the leapfrog method with a 2 fs time

667 step, and the LINCS algorithm was used to constrain bond lengths ${ }^{65}$. Electrostatic

668 interactions were computed using the Particle-Mesh-Ewald (PME) algorithm ${ }^{66}$ and the real-

669 space sum was cut off at $12 \AA$. Van der Waals interactions were switched off between $10 \AA$

670 and $12 \AA$. The neighbour list was updated every 10 steps. Simulations were performed using

671 conditions of constant temperature $(310 \mathrm{~K})$ and pressure $(1 \mathrm{~atm})$ via the Bussi thermostat ${ }^{67}$,

672 and semi-isotropic pressure-coupling using the Parrinello-Rahman barostat ${ }^{68}$ with a coupling

673 constant of $5 \mathrm{ps,} \mathrm{under} \mathrm{periodic-boundary} \mathrm{conditions.} \mathrm{Visual} \mathrm{analyses} \mathrm{and} \mathrm{preparation} \mathrm{of}$

674 molecular graphics from the simulation trajectories were performed using VMD ${ }^{69}$. Further

675 analysis was performed using GROMACS and graphs were prepared with Grace

676 (http://plasma-gate.weizmann.ac.il/Grace/). Simulations were performed using the Darwin

677 Supercomputer of the University of Cambridge High Performance Computing Service.

679 Acknowledgements

680

We thank Evelyne Richet and Jenny-Lee Thomassin for insightful comments and

682 critical reading of the manuscript. We thank Cesar Valencia and Jenny-Lee Thomassin for

683 help with IF data processing and statistical analysis, Mariette Bonnet for help and advice, and

684 Stéphane Romero for stimulating discussions about biological fibres. We are grateful to all

685 members of the Laboratory of Intercellular Communication and Microbial Infections and of

686 the Laboratory for Macromolecular Systems and Signalling for helpful discussions and 
friendly support. We thank Gouzel Karimova and Daniel Ladant for the strains, plasmids and

688 advice concerning the BAC2H analysis. This work was funded by the ANR FiberSpace grant

$689 \mathrm{~N}^{\circ}$ ANR-14-CE09-0004. Javier Santos-Moreno was funded by a fellowship from the Basque

690 Government; Alexandra East was supported by an EPSRC PhD studentship. The

691 computational work benefited from use of the Darwin Supercomputer of the University of

692 Cambridge High Performance Computing Service (http://www.hpc.cam.ac.uk/), provided by

693 Dell Inc. using Strategic Research, Infrastructure Funding from the Higher Education Funding

694 Council for England. The authors state that they have no conflict of interest.

695

\section{References}

697 1. Berry, J. L. \& Pelicic, V. (2015). Exceptionally widespread nanomachines 698 composed of type IV pilins: the prokaryotic Swiss Army knives. FEMS Microbiology 699 Reviews 39, 134-54.

$7002 . \quad J a r r e l l$, K. F. \& Albers, S. V. (2012). The archaellum: an old motility structure with 701 a new name. Trends in Microbiology 20, 307-12.

702 3. Makarova, K. S., Koonin, E. V. \& Albers, S. V. (2016). Diversity and Evolution of 703 Type IV pili Systems in Archaea. Front Microbiol 7, 667.

704 4. Pohlschroder, M. \& Esquivel, R. N. (2015). Archaeal type IV pili and their 705 involvement in biofilm formation. Front Microbiol 6, 190.

706 5. Melville, S. \& Craig, L. (2013). Type IV pili in Gram-positive bacteria. Microbiology 707 and Molecular Biology Reviews 77, 323-41.

708 6. Korotkov, K. V., Sandkvist, M. \& Hol, W. G. (2012). The type II secretion system: 709 biogenesis, molecular architecture and mechanism. Nat Rev Microbiol 10, 336-51.

$7107 . \quad$ Campos, M., Cisneros, D. A., Nivaskumar, M. \& Francetic, O. (2013). The type II 711 secretion system - a dynamic fiber assembly nanomachine. Res Microbiol 164, 545-55.

712 8. Nivaskumar, M. \& Francetic, O. (2014). Type II secretion system: A magic 713 beanstalk or a protein escalator. Biochim Biophys Acta.

$7149 . \quad$ Strom, M. S. \& Lory, S. (1991). Amino acid substitutions in pilin of Pseudomonas 715 aeruginosa. Effect on leader peptide cleavage, amino-terminal methylation, and pilus 716 assembly.J Biol Chem 266, 1656-64.

717 10. Strom, M. S., Nunn, D. N. \& Lory, S. (1993). A single bifunctional enzyme, PilD, 718 catalyzes cleavage and N-methylation of proteins belonging to the type IV pilin family. 
721 11. Strom, M. S., Bergman, P. \& Lory, S. (1993). Identification of active-site cysteines 722 in the conserved domain of PilD, the bifunctional type IV pilin leader peptidase/N723 methyltransferase of Pseudomonas aeruginosa. Journal of Biological Chemistry 268, 724 15788-94.

725 12. Chang, Y. W., Rettberg, L. A., Treuner-Lange, A., Iwasa, J., Sogaard-Andersen, L. \& 726 Jensen, G. J. (2016). Architecture of the type IVa pilus machine. Science 351, aad2001.

727 13. Possot, O., d'Enfert, C., Reyss, I. \& Pugsley, A. P. (1992). Pullulanase secretion in 728 Escherichia coli K-12 requires a cytoplasmic protein and a putative polytopic 729 cytoplasmic membrane protein. Molecular Microbiology 6, 95-105.

730 14. Py, B., Loiseau, L. \& Barras, F. (2001). An inner membrane platform in the type II 731 secretion machinery of Gram-negative bacteria. EMBO Rep 2, 244-8.

732 15. Chami, M., Guilvout, I., Gregorini, M., Remigy, H. W., Muller, S. A., Valerio, M., Engel, 733 A., Pugsley, A. P. \& Bayan, N. (2005). Structural insights into the secretin PulD and its 734 trypsin-resistant core. Journal of Biological Chemistry 280, 37732-41.

735 16. Sauvonnet, N., Vignon, G., Pugsley, A. P. \& Gounon, P. (2000). Pilus formation and 736 protein secretion by the same machinery in Escherichia coli. EMBO Journal 19, 2221-8.

737 17. Durand, E., Bernadac, A., Ball, G., Lazdunski, A., Sturgis, J. N. \& Filloux, A. (2003). 738 Type II protein secretion in Pseudomonas aeruginosa: the pseudopilus is a multifibrillar 739 and adhesive structure. Journal of Bacteriology 185, 2749-58.

740 18. Gray, M. D., Bagdasarian, M., Hol, W. G. \& Sandkvist, M. (2011). In vivo cross741 linking of EpsG to EpsL suggests a role for EpsL as an ATPase-pseudopilin coupling 742 protein in the Type II secretion system of Vibrio cholerae. Mol Microbiol 79, 786-98.

743 19. Sandkvist, M., Hough, L. P., Bagdasarian, M. M. \& Bagdasarian, M. (1999). Direct 744 interaction of the EpsL and EpsM proteins of the general secretion apparatus in Vibrio 745 cholerae. Journal of Bacteriology 181, 3129-35.

746 20. Abendroth, J., Murphy, P., Sandkvist, M., Bagdasarian, M. \& Hol, W. G. (2005). The 747 X-ray structure of the type II secretion system complex formed by the N-terminal 748 domain of EpsE and the cytoplasmic domain of EpsL of Vibrio cholerae. J Mol Biol 348, $749845-55$.

750 21. Camberg, J. L., Johnson, T. L., Patrick, M., Abendroth, J., Hol, W. G. \& Sandkvist, M. 751 (2007). Synergistic stimulation of EpsE ATP hydrolysis by EpsL and acidic 752 phospholipids. EMBO J 26, 19-27.

753 22. Michel, G., Bleves, S., Ball, G., Lazdunski, A. \& Filloux, A. (1998). Mutual 754 stabilization of the XcpZ and XcpY components of the secretory apparatus in 755 Pseudomonas aeruginosa. Microbiology 144 ( Pt 12), 3379-86. 
23. Johnson, T. L., Scott, M. E. \& Sandkvist, M. (2007). Mapping critical interactive sites within the periplasmic domain of the Vibrio cholerae type II secretion protein EpsM. Journal of Bacteriology 189, 9082-9.

24. Possot, O. M., Vignon, G., Bomchil, N., Ebel, F. \& Pugsley, A. P. (2000). Multiple interactions between pullulanase secreton components involved in stabilization and cytoplasmic membrane association of PulE. J Bacteriol 182, 2142-52.

25. Nivaskumar, M., Santos-Moreno, J., Malosse, C., Nadeau, N., Chamot-Rooke, J., Tran Van Nhieu, G. \& Francetic, O. (2016). Pseudopilin residue E5 is essential for recruitment by the type 2 secretion system assembly platform. Molecular Microbiology 101, 924-41.

26. Georgiadou, M., Castagnini, M., Karimova, G., Ladant, D. \& Pelicic, V. (2012). Largescale study of the interactions between proteins involved in type IV pilus biology in Neisseria meningitidis: characterization of a subcomplex involved in pilus assembly. Mol Microbiol 84, 857-73.

27. Karuppiah, V., Collins, R. F., Thistlethwaite, A., Gao, Y. \& Derrick, J. P. (2013). Structure and assembly of an inner membrane platform for initiation of type IV pilus biogenesis. Proc Natl Acad Sci U S A 110, E4638-47.

28. Tammam, S., Sampaleanu, L. M., Koo, J., Manoharan, K., Daubaras, M., Burrows, L. L. \& Howell, P. L. (2013). PilMNOPQ from the Pseudomonas aeruginosa type IV pilus system form a transenvelope protein interaction network that interacts with PilA. $J$ Bacteriol 195, 2126-35.

29. Pasloske, B. L., Scraba, D. G. \& Paranchych, W. (1989). Assembly of mutant pilins in Pseudomonas aeruginosa: formation of pili composed of heterologous subunits. Journal of Bacteriology 171, 2142-7.

30. Aas, F. E., Winther-Larsen, H. C., Wolfgang, M., Frye, S., Lovold, C., Roos, N., van Putten, J. P. \& Koomey, M. (2007). Substitutions in the N-terminal alpha helical spine of Neisseria gonorrhoeae pilin affect Type IV pilus assembly, dynamics and associated functions. Molecular Microbiology 63, 69-85.

31. Pugsley, A. P. (1993). Processing and methylation of PuIG, a pilin-like component of the general secretory pathway of Klebsiella oxytoca. Mol Microbiol 9, 295-308.

32. Vignon, G., Kohler, R., Larquet, E., Giroux, S., Prevost, M. C., Roux, P. \& Pugsley, A. P. (2003). Type IV-like pili formed by the type II secreton: specificity, composition, bundling, polar localization, and surface presentation of peptides. J Bacteriol 185, 341628.

33. Campos, M., Nilges, M., Cisneros, D. A. \& Francetic, O. (2010). Detailed structural and assembly model of the type II secretion pilus from sparse data. Proc Natl Acad Sci U $S$ A 107, 13081-6.

34. Nivaskumar, M., Bouvier, G., Campos, M., Nadeau, N., Yu, X., Egelman, E. H., Nilges, M. \& Francetic, O. (2014). Distinct docking and stabilization steps of the pseudopilus conformational transition path suggest rotational assembly of type IV pilus-like fibers. Structure 22, 685-96. 
35. Cisneros, D. A., Bond, P. J., Pugsley, A. P., Campos, M. \& Francetic, O. (2012). Minor pseudopilin self-assembly primes type II secretion pseudopilus elongation. EMBO J 31, 1041-53.

36. Karimova, G., Pidoux, J., Ullmann, A. \& Ladant, D. (1998). A bacterial two-hybrid system based on a reconstituted signal transduction pathway. Proc Natl Acad Sci U S A 95, 5752-6.

37. Dautin, N., Karimova, G., Ullmann, A. \& Ladant, D. (2000). Sensitive genetic screen for protease activity based on a cyclic AMP signaling cascade in Escherichia coli. Journal of Bacteriology 182, 7060-6.

38. Durand, E., Michel, G., Voulhoux, R., Kurner, J., Bernadac, A. \& Filloux, A. (2005). XcpX controls biogenesis of the Pseudomonas aeruginosa XcpT-containing pseudopilus. J Biol Chem 280, 31378-89.

39. Lybarger, S. R., Johnson, T. L., Gray, M. D., Sikora, A. E. \& Sandkvist, M. (2009). Docking and assembly of the type II secretion complex of Vibrio cholerae. Journal of Bacteriology 191, 3149-61.

40. Buddelmeijer, N., Krehenbrink, M., Pecorari, F. \& Pugsley, A. P. (2009). Type II secretion system secretin PulD localizes in clusters in the Escherichia coli outer membrane. Journal of Bacteriology 191, 161-8.

41. Kolappan, S., Coureuil, M., Yu, X., Nassif, X., Egelman, E. H. \& Craig, L. (2016). Structure of the Neisseria meningitidis Type IV pilus. Nat Commun 7, 13015.

42. Craig, L., Volkmann, N., Arvai, A. S., Pique, M. E., Yeager, M., Egelman, E. H. \& Tainer, J. A. (2006). Type IV pilus structure by cryo-electron microscopy and crystallography: implications for pilus assembly and functions. Mol Cell 23, 651-62.

43. Craig, L., Taylor, R. K., Pique, M. E., Adair, B. D., Arvai, A. S., Singh, M., Lloyd, S. J., Shin, D. S., Getzoff, E. D., Yeager, M., Forest, K. T. \& Tainer, J. A. (2003). Type IV pilin structure and assembly: X-ray and EM analyses of Vibrio cholerae toxin-coregulated pilus and Pseudomonas aeruginosa PAK pilin. Molecular Cell 11, 1139-50.

44. Lemkul, J. A. \& Bevan, D. R. (2011). Characterization of interactions between PilA from Pseudomonas aeruginosa strain K and a model membrane. J Phys Chem B 115, 8004-8.

45. Andersson, H. \& von Heijne, G. (1994). Membrane protein topology: effects of delta $\mathrm{mu} \mathrm{H}+$ on the translocation of charged residues explain the 'positive inside' rule. EMBO Journal 13, 2267-72.

46. Pasloske, B. L. \& Paranchych, W. (1988). The expression of mutant pilins in Pseudomonas aeruginosa: fifth position glutamate affects pilin methylation. Mol Microbiol 2, 489-95.

47. Thomas, N. A., Bardy, S. L. \& Jarrell, K. F. (2001). The archaeal flagellum: a different kind of prokaryotic motility structure. FEMS Microbiology Reviews 25, 147-74. 
834 48. Albers, S. V. \& Meyer, B. H. (2011). The archaeal cell envelope. Nat Rev Microbiol $8359,414-26$.

836 49. Ota, A., Gmajner, D., Sentjurc, M. \& Ulrih, N. P. (2012). Effect of growth medium pH 837 of Aeropyrum pernix on structural properties and fluidity of archaeosomes. Archaea $8382012,285152$.

839 50. Trincone, A., Nicolaus, B., Palmieri, G., De Rosa, M., Huber, R., Huber, G., Stetter, K. 840 0. \& Gambacorta, A. (1992). Distribution of complex and core lipids within new 841 hyperthermophilic members of the Archaea domain. Systematic and Applied 842 Microbiology 15, 11-17.

843 51. Lallemand, M., Login, F. H., Guschinskaya, N., Pineau, C., Effantin, G., Robert, X. \& 844 Shevchik, V. E. (2013). Dynamic interplay between the periplasmic and transmembrane 845 domains of GspL and GspM in the type II secretion system. PLoS One 8, e79562.

846 52. Leighton, T. L., Dayalani, N., Sampaleanu, L. M., Howell, P. L. \& Burrows, L. L. 847 (2015). Novel Role for PilNO in Type IV Pilus Retraction Revealed by Alignment 848 Subcomplex Mutations. Journal of Bacteriology 197, 2229-38.

849 53. Pardee, A. B., Jacob, F. \& Monod, J. (1959). The genetic control and cytoplaslic 850 expression of "inducibility" in the synthesis of $\beta$-galactosidase by E. coli. J Mol Biol 1, 851 165-178.

852 54. Pugsley, A. P., Bayan, N. \& Sauvonnet, N. (2001). Disulfide bond formation in 853 secreton component PulK provides a possible explanation for the role of DsbA in 854 pullulanase secretion. Journal of Bacteriology 183, 1312-9.

855 55. Kohler, R., Schafer, K., Muller, S., Vignon, G., Diederichs, K., Philippsen, A., Ringler, 856 P., Pugsley, A. P., Engel, A. \& Welte, W. (2004). Structure and assembly of the pseudopilin 857 PulG. Mol Microbiol 54, 647-64.

858 56. Bartolome, B., Jubete, Y., Martinez, E. \& de la Cruz, F. (1991). Construction and 859 properties of a family of pACYC184-derived cloning vectors compatible with pBR322 860 and its derivatives. Gene 102, 75-8.

861 57. Spratt, B. G., Hedge, P. J., te Heesen, S., Edelman, A. \& Broome-Smith, J. K. (1986). 862 Kanamycin-resistant vectors that are analogues of plasmids pUC8, pUC9, pEMBL8 and 863 pEMBL9. Gene 41, 337-42.

864 58. Schagger, H. \& von Jagow, G. (1987). Tricine-sodium dodecyl sulfate865 polyacrylamide gel electrophoresis for the separation of proteins in the range from 1 to 866100 kDa. Analytical Biochemistry 166, 368-79.

867 59. Abràmoff, M. D., Magalhães, P. J. \& Ram, S. J. (2004). Image processing with 868 Image J. Biophoton Int 11, 36-43.

869 60. Miller, J. H. (1972). Experiments in molecular genetics, Cold Spring Harbor 870 Laboratory, Cold Spring Harbor, New York. 
871 61. Wolf, M. G., Hoefling, M., Aponte-Santamaria, C., Grubmuller, H. \& Groenhof, G. 872 (2010). g_membed: Efficient insertion of a membrane protein into an equilibrated lipid 873 bilayer with minimal perturbation. J Comput Chem 31, 2169-74.

874 62. Hess, B., Kutzner, C., van der Spoel, D. \& Lindahl, E. (2008). GROMACS 4: 875 Algorithms for Highly Efficient, Load-Balanced, and Scalable Molecular Simulation. J 876 Chem Theory Comput 4, 435-47.

877 63. Bjelkmar, P., Larsson, P., Cuendet, M. A., Hess, B. \& Lindahl, E. (2010). 878 Implementation of the CHARMM Force Field in GROMACS: Analysis of Protein Stability 879 Effects from Correction Maps, Virtual Interaction Sites, and Water Models. J Chem Theory 880 Comput 6, 459-66.

881 64. MacKerell, A. D., Bashford, D., Bellott, M., Dunbrack, R. L., Evanseck, J. D., Field, M. 882 J., Fischer, S., Gao, J., Guo, H., Ha, S., Joseph-McCarthy, D., Kuchnir, L., Kuczera, K., Lau, F. 883 T., Mattos, C., Michnick, S., Ngo, T., Nguyen, D. T., Prodhom, B., Reiher, W. E., Roux, B., 884 Schlenkrich, M., Smith, J. C., Stote, R., Straub, J., Watanabe, M., Wiorkiewicz-Kuczera, J., 885 Yin, D. \& Karplus, M. (1998). All-atom empirical potential for molecular modeling and 886 dynamics studies of proteins. J Phys Chem B 102, 3586-616.

887 65. Hess, B. (1997). Periodic patterns in biochemical reactions. Quarterly Reviews of 888 Biophysics 30, 121-76.

889 66. Essmann, U., Perera, L., Berkowitz, M. L., Darden, T., Lee, H. \& Pedersen, L. G. 890 (1995). A smooth particle mesh Ewald potential. Journal of Chemical Physics 103, 85778918592.

892 67. Bussi, G., Donadio, D. \& Parrinello, M. (2007). Canonical sampling through 893 velocity rescaling. Journal of Chemical Physics 126, 014101.

894 68. Parrinello, M. \& Rahman, A. (1981). Polymorphic transitions in single crystals: A 895 new molecular dynamics method. J Appl Phys 52, 7182-7190.

896 69. Humphrey, W., Dalke, A. \& Schulten, K. (1996). VMD: visual molecular dynamics. 897 Journal of Molecular Graphics 14, 33-8, 27-8.

898 70. Waterhouse, A. M., Procter, J. B., Martin, D. M., Clamp, M. \& Barton, G. J. (2009). 899 Jalview Version 2-a multiple sequence alignment editor and analysis workbench. 900 Bioinformatics 25, 1189-91.

901

902 

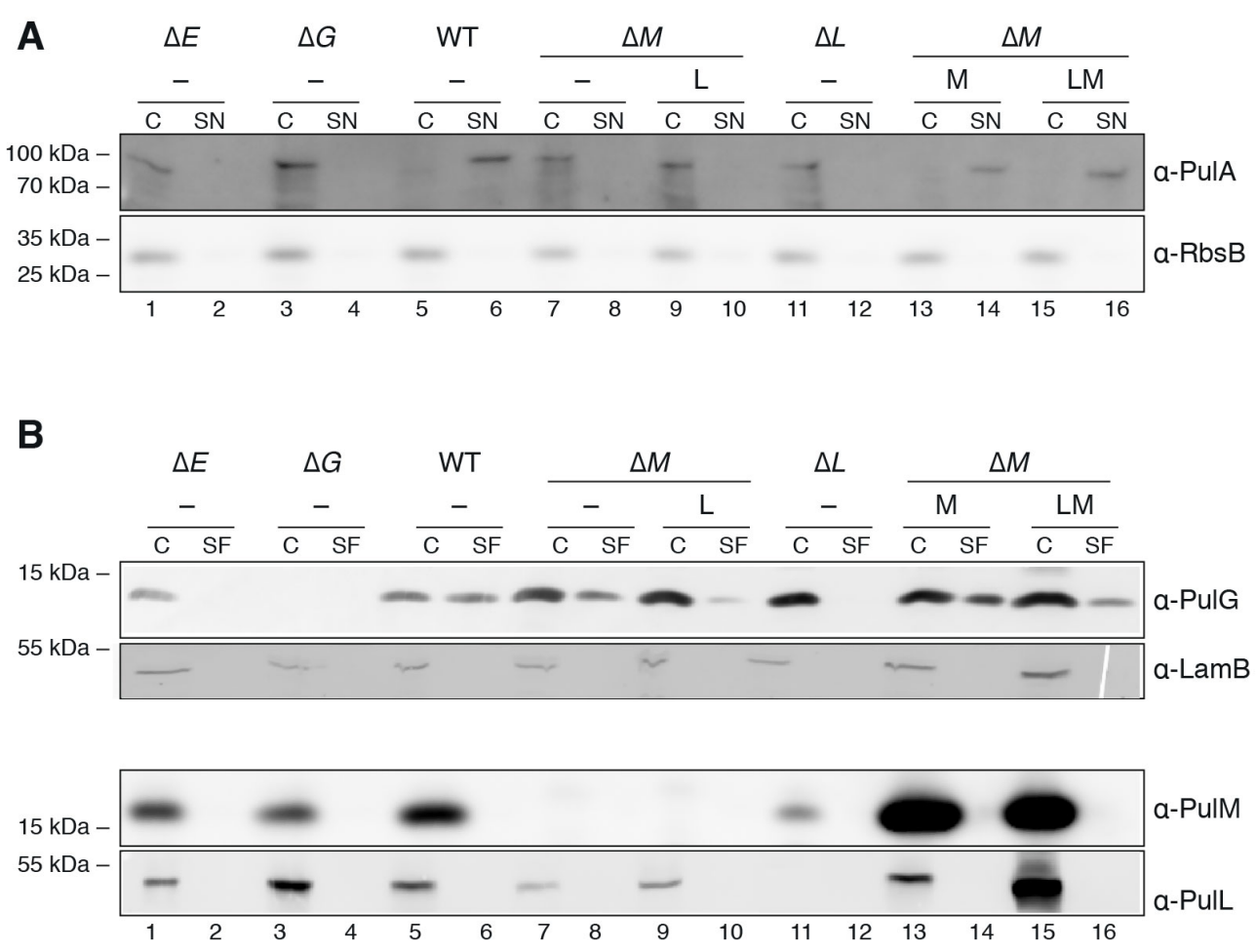

C

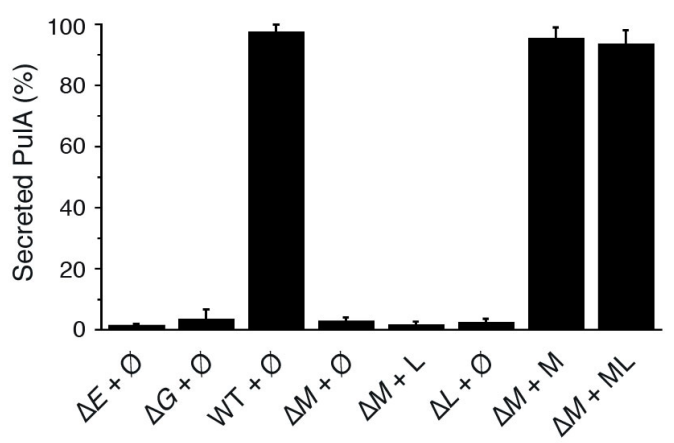

D

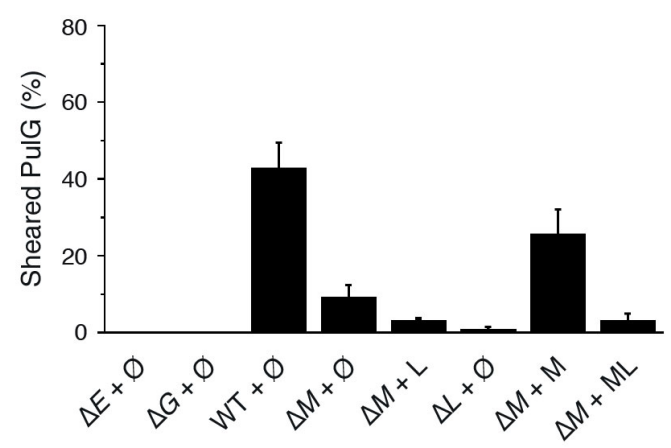

Fig. 1. The role of PulM in T2SS function

907 A. PulA secretion assay in near-chromosomal expression conditions using E. coli PAP5299

908 co-transformed with pCHAP8185 (all pul genes, WT) or its derivatives containing single

909 nonpolar pul gene deletions as indicated by a single letter code: $\Delta E$ (pCHAP8200), $\Delta G$

910 (pCHAP8184), $\Delta M$ (pCHAP8496), or $\Delta L$ (pCHAP8251); and with compatible pSU19 (-) or

911 its derivatives carrying indicated pul genes: L (pCHAP8258), M (pCHAP1353) or LM

912 (pCHAP8843). The amount of pullulanase PulA in $0.015 \mathrm{OD}_{600}$ units of cell extracts (C) and 
913 culture supernatants (SN) was assessed by Western blot. Immunodetection of the periplasmic

914 ribose-binding protein $\mathrm{RbsB}$ in $0.03 \mathrm{OD}_{600}$ units served as a lysis control. Molecular weight $915(\mathrm{Mw})$ markers and lane numbers are shown.

916 B. PulG pilus assembly assay of E. coli PAP7460 overexpressing the pul genes from the same 917 plasmids as in (A). Cell (C) and sheared pili (SF) fractions from an equivalent of $0.05 \mathrm{OD}_{600}$ 918 units were separated on Tris-Tricin SDS-PAGE, transferred onto nitrocellulose membranes 919 and probed with antibodies against PulG, LamB, PulM and PulL. Mw markers and lane 920 numbers are shown.

921 C. Quantification of the percentage of secreted PulA (mean + standard deviation - s.d.) from 922 three independent experiments as the one in (A). Ø indicates empty vector.

923 D. Quantification of the percentage of sheared PulG (mean + s.d.) from three independent 924 experiments like the one in (B). Ø, empty vector. 
A

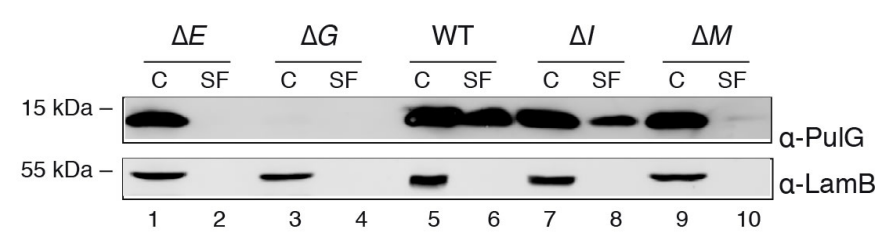

C

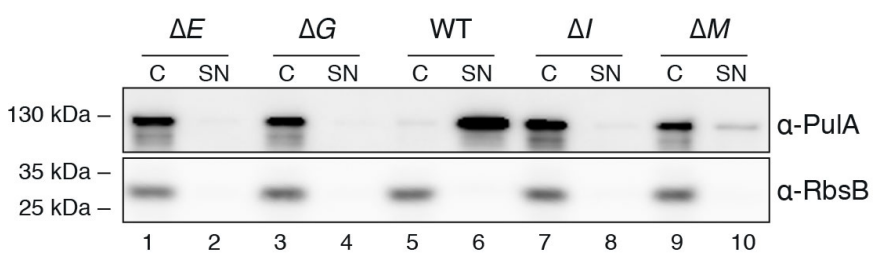

E

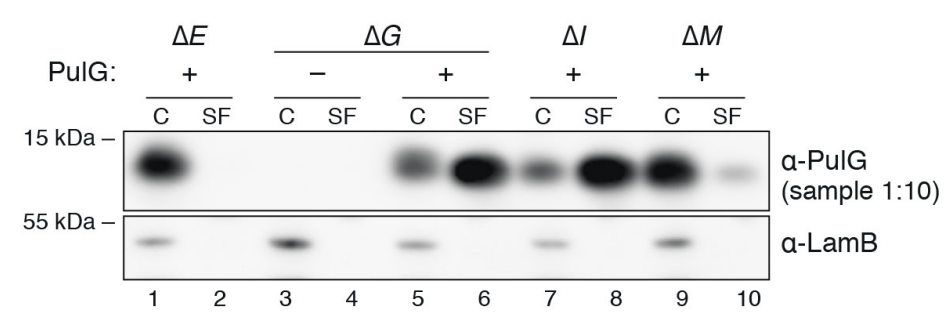

B

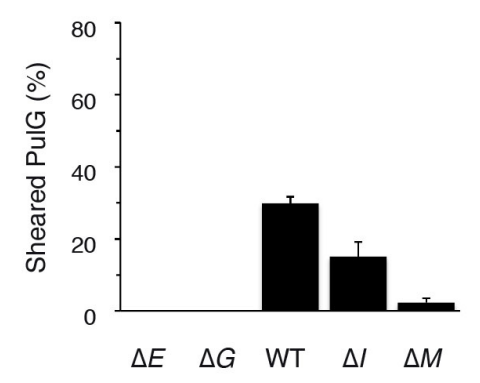

D

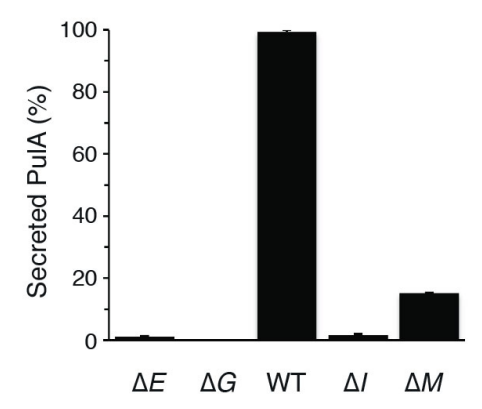

$\mathbf{F}$

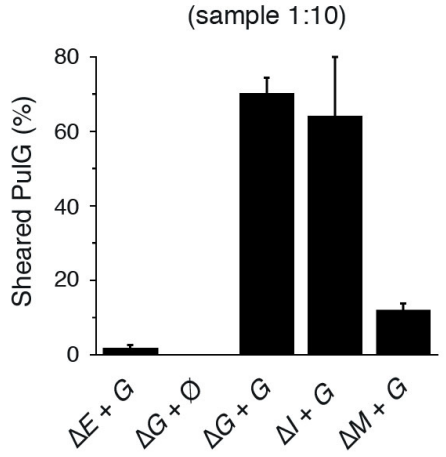

926

927 Fig. 2. Characterisation of $\mathbf{A p u l M}$ mutant function under conditions of pul gene 928 overexpression.

929 Shearing (A and E) and secretion (C) assays and the corresponding quantifications of the

930 percentage of sheared PulG (mean + s.d.) (B and F) or secreted PulA (D) in three independent 931 experiments.

932 A. Immunodetection of PulG $0.05 \mathrm{OD}_{600}$ units of cell (C) and sheared fractions (SF) of E. coli

933 PAP7460 transformed with plasmid pCHAP8185 containing all pul genes (WT) or its single

934 gene deletion derivatives: $\Delta E$ (pCHAP8200), $\Delta G$ (pCHAP8184), $\Delta I$ (pCHAP8218) and $\Delta M$

935 (pCHAP8496). LamB immuno-detection is shown as control. Mw markers are indicated on 936 the left. 
937 B. Percent of sheared PulG (mean + s.d.) from three independent experiments like the one 938 shown in $(\mathrm{A})$.

939 C. PulA secretion assay using transformed PAP7460 derivatives as in (A). PulA was detected 940 by Western blot in $0.02 \mathrm{OD}_{600}$ units of cell extracts (C) and culture supernatants (SN).

941 Immunodetection of periplasmic RbsB served as a lysis control. Mw markers and lane 942 numbers are shown.

943 D. Quantification of the percentage of secreted PulA (mean + s.d.) from three independent 944 experiments like the one in (C).

945 E. Effect of pulG overexpression on pseudopilus assembly. Top: Anti-PulG immunoblot of $9460.005 \mathrm{OD}_{600}$ units ("sample 1:10") of cell (C) and sheared fractions (SF) of bacteria 947 transformed with pSU19 (-) or its derivative pCHAP8658 encoding PulG (+) and indicated $948 p u l$ gene expression plasmids containing single gene deletions, as in (A): $\Delta E$ (pCHAP8200), $949 \Delta G$ (pCHAP8184), $\Delta I$ (pCHAP8218), or $\Delta M$ (pCHAP8496). Bottom: Anti-LamB control of a 95010 -fold concentrated sample (i.e. $0.05 \mathrm{OD}_{600}$ units). The position of the $\mathrm{Mw}$ markers is 951 indicated.

952 F. Percent (mean + s.d.) of PulG in the sheared fraction from three independent experiments 953 as in (E), using $0.005 \mathrm{OD}_{600}$ units (“sample 1:10”). Ø, empty vector. 
A
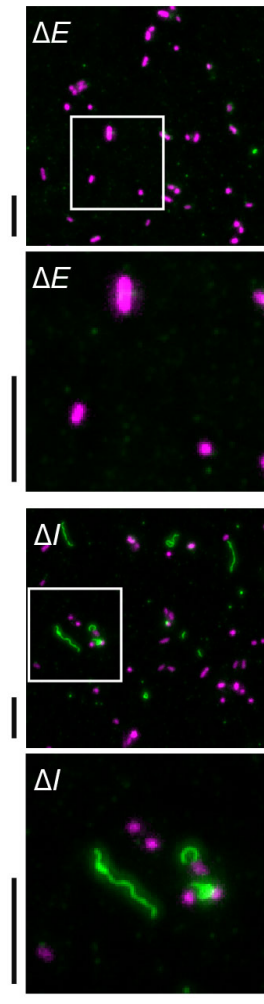
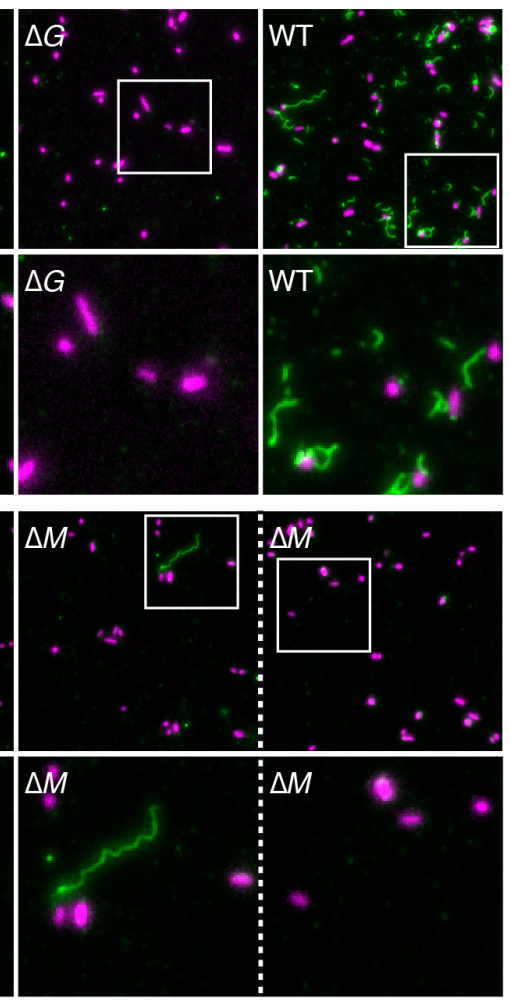

B

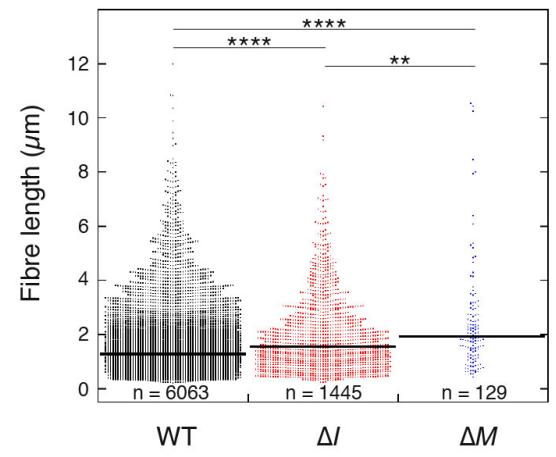

C

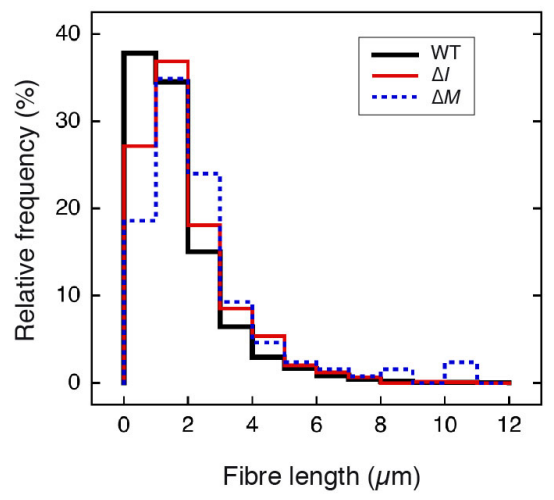

955

956

957 Bacteria (magenta) and PulG fibres (green) were labelled and examined by IF (Materials and 958 Methods).

959 A. Representative fluorescence micrographs. WT: wild-type; $\Delta E: \Delta p u l E ; \Delta G: \Delta p u l G ; \Delta I$ :

$960 \Delta p u l I ; \Delta M: \Delta p u l M$. A 2.6-fold magnified inset is shown below each image. An additional

961 panel is shown for $\Delta p u l M$ to illustrate the absence of fibres in the majority of the fields. Scale

962 bars $=5 \mu \mathrm{m}$.

963 B. Dot-plot representing the length of individual fibres (dots) scored for 12864, 13062 and

96414786 bacteria (for WT, $\Delta p u l I$ and $\triangle p u l M$ respectively) in three independent experiments. On

965 average, $0.47 \pm 0.09$ (mean \pm s.d.,) fibres per bacterium were found in WT, $0.11 \pm 0.2$ in

$966 \Delta p u l I$ and $0.009 \pm 0.003$ in $\Delta p u l M$ strain). The median fibre length values, shown as

967 horizontal black lines, are $1.2 \pm 0.6 \mu \mathrm{m}$ (median \pm m.a.d., median absolute deviation) for WT,

$9681.5 \pm 0.7 \mu \mathrm{m}$ for $\Delta p u l I$ strain and $1.8 \pm 0.7 \mu \mathrm{m}$ for $\Delta p u l M$ strain. Dunn's test, $* * \mathrm{p}<0.01$, $969 * * * * \mathrm{p}<0.0001$.

970 C. Relative frequency distribution of fibre length. Bin size $=1 \mu \mathrm{m}$. 


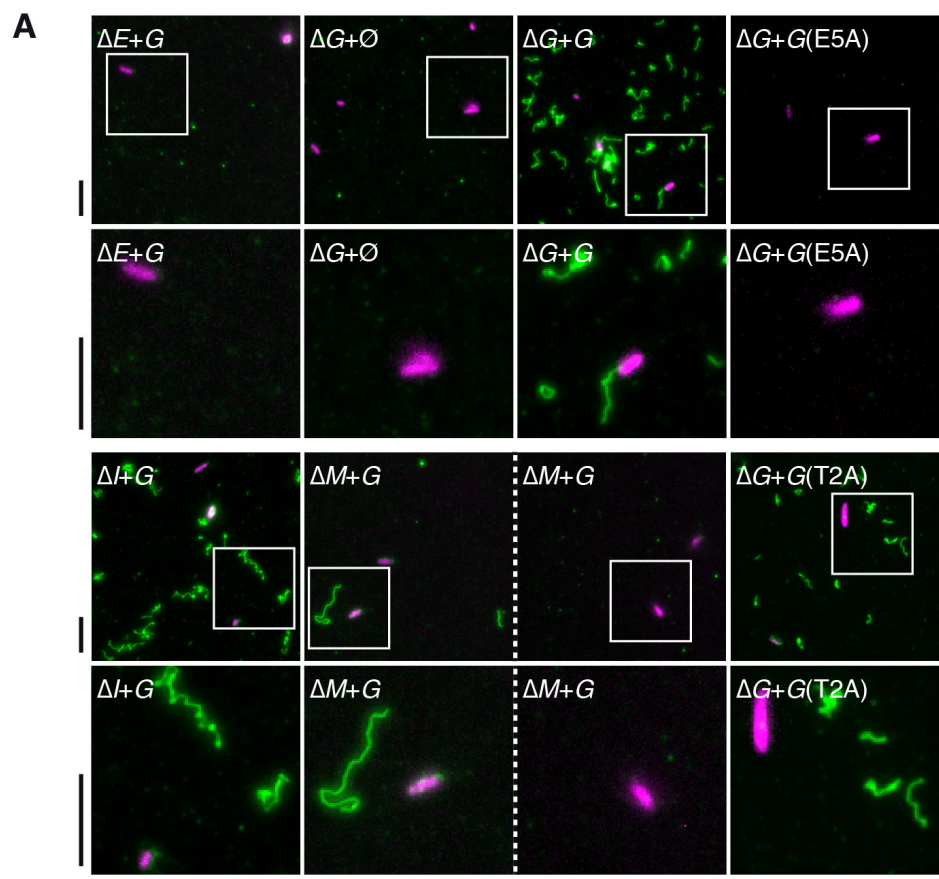

B

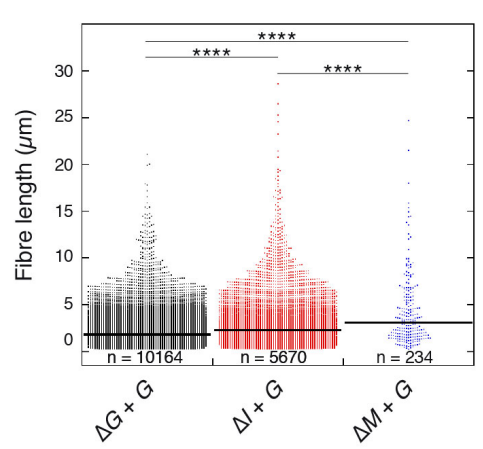

C

D
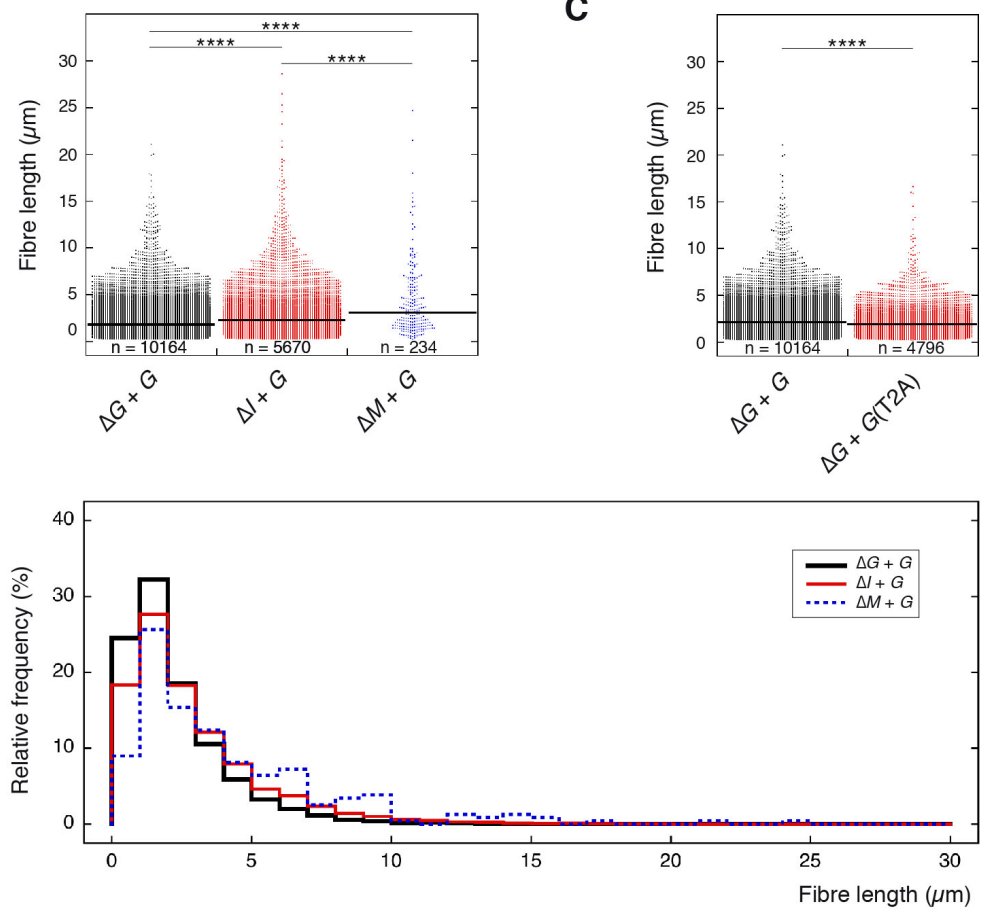

E

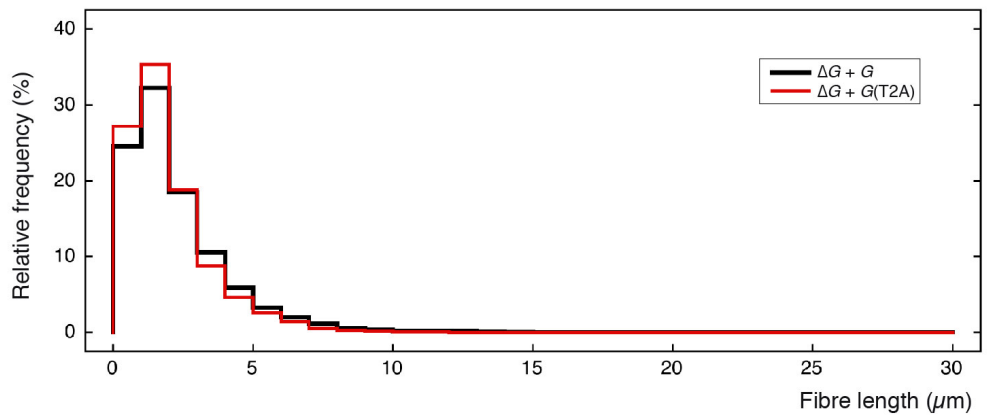




\section{Fig. 4. The effect of PulG overproduction on T2S pilus assembly.}

973 A. IF microscopy of the piliation ability of $\Delta p u l M+p u l G$ and $\Delta p u l G+p u l G(\mathrm{~T} 2 \mathrm{~A})$. Bacteria,

974 magenta; fibres, green. A 2.6-fold magnified inset is shown below each image. An extra panel

975 is shown for $\Delta p u l M+p u l G$ to illustrate the absence of fibres in most of the fields. The scale

976 bars $(5 \mu \mathrm{m})$ are shown on the left.

977 B and C. Dot-plots representing the length of individual fibres (dots) scored for 1342, 1190,

9781597 and 1460 bacteria (for $\Delta p u l G+p u l G, \Delta p u l I+p u l G, \Delta p u l M+p u l G$ and $\Delta p u l G+$

$979 \mathrm{pulG}(T 2 A)$, respectively) in three independent experiments. The number of fibres/bacterium

980 (expressed as mean \pm s.d) were $7.64 \pm 1.49$ (for strain $\Delta p u l G+p u l G$ ); $4.7 \pm 0.6$ (for strain

$981 \Delta p u l I+p u l G$ ); $0.15 \pm 0.06$ (for strain $\Delta p u l M+p u l G$ ); and $3.73 \pm 1.68$ (for strain $\Delta p u l G+$

982 pulG(T2A)). The median values of fibre length are shown as a horizontal black lines: for

$983 \Delta p u l G+p u l G, 1.7 \pm 0.8 \mu \mathrm{m}$ (median \pm m.a.d.); for $\Delta p u l I+p u l G, 2.2 \pm 1.1 \mu \mathrm{m}$; for $\Delta p u l M+$

$984 p u l G, 3.0 \pm 1.6 \mu \mathrm{m}$ and for $\Delta p u l G+p u l G(T 2 A), 1.6 \pm 0.7 \mu \mathrm{m}$. Dunn's test, $* * * * \mathrm{p}<0.0001$.

$985 \mathrm{D}$ and E. Relative frequency distributions of fibre length. Bin $=1 \mu \mathrm{m}$. 
A \begin{tabular}{r|l} 
T2SS & $\begin{array}{l}\text { Klebsiella oxytoca } \\
\text { Vibrio cholerae } \\
\text { Escherichia coli } \\
\text { Pseudomonas aeruginosa } \\
\text { Dickeya dadantii } \\
\text { Aeromonas hydrophila }\end{array}$ \\
$\mathrm{T} 4 \mathrm{P}$ & $\begin{array}{l}\text { Pseudomonas aeruginosa } \\
\text { Neisseria gonorrhoeae }\end{array}$
\end{tabular}

B

PulG
EspG
GspG
XcpT
OutG
ExeG
PilA
Pile

$-5 \ldots \nabla_{1} \ldots 5 \ldots 10 \ldots \ldots 20 \ldots \ldots 30$ -- MQRORG FTLLEIMVVIVILGVLASLVVPNLMGNKEK MKKMRKQTG FTLLEVMVVVVILGILASFVVPNLLGNKE MRATDKQRG FTLLEIMVVIVI IGVLASLVVPNLMGNKE -MQRRQQSG FTLIEIMVVVVILGILAALVVPQVMSRPD --MERRQRG FTLLEIMVVIVILGVLASLVVPNLMGNKE -MQKRRQSG FTLLEVMVVIVILGILASLVVPNLMGNKE --MKAQKG FTLIELMIVVAIIGILAAIAIPQYQDYTA --MNTLQKG FTLIELMIVIAIVGILAAVALPAYQDYTA

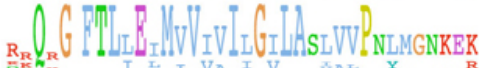
MM+MRRQRG $\frac{1}{\text { FTLLEIMVVIVILGILASLVVPNLMGNKEK }}$

$-5 \ldots \nabla_{1} \ldots 5 \ldots 10$

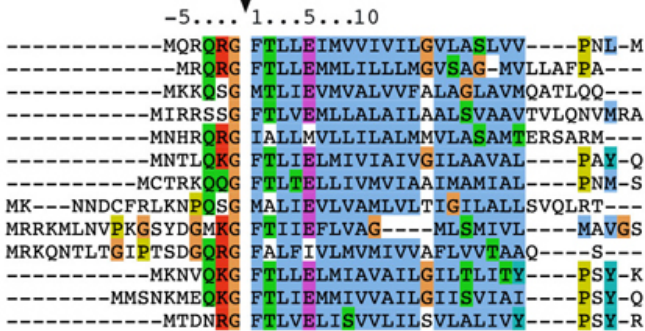

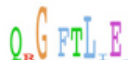

MR----N----M++RQRG FTLIE+++VLAILGILALIALQV-LPSY-+

C

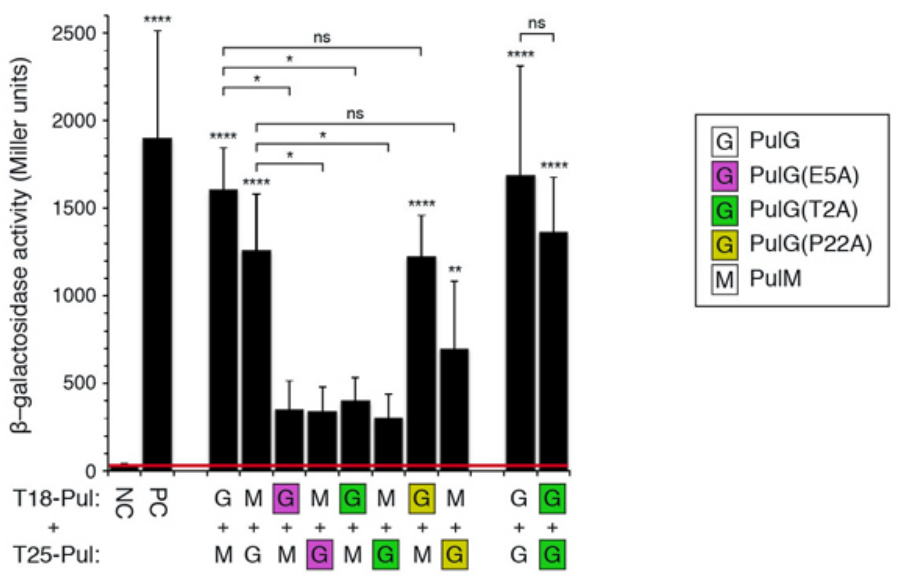

D

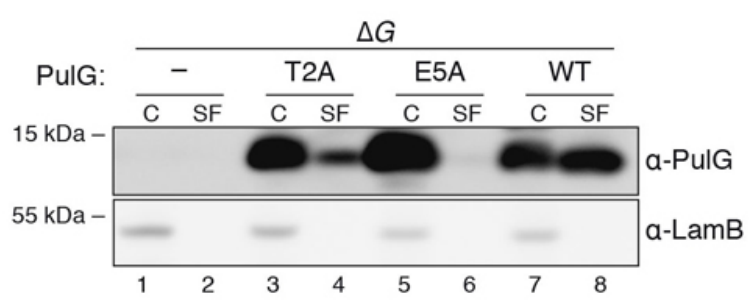

$\mathbf{F}$

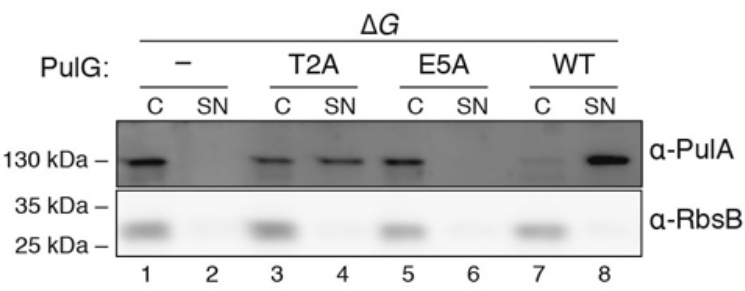

E

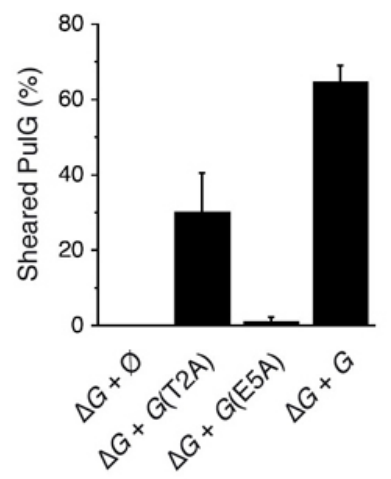

G

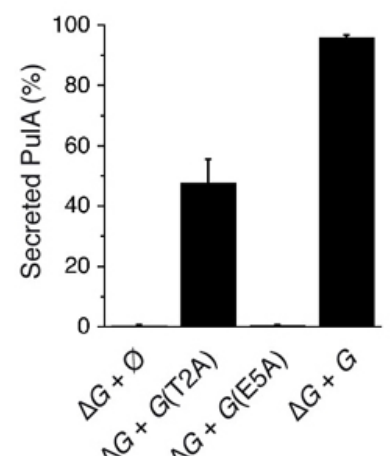


987 Fig. 5. PulG ${ }^{\mathrm{T} 2 \mathrm{~A}}$ variant shows defective interaction with PulM, piliation and secretion.

988 A. Protein sequence alignment of the N-terminal segments of major T2SS pseudopilin and 989 T4P subunits from the indicated bacterial species. The consensus sequence is indicated below 990 and arrowheads indicate the prepilin peptidase cleavage site.

991 B. Sequence alignment of major and minor pseudopilins from the Pul T2SS of Klebsiella 992 oxytoca (top) and major and minor T4P pilins from Neisseria meningitidis (bottom). The 993 arrowheads indicate the prepilin peptidase cleavage site. Alignments were generated using 994 Jalview2 software ${ }^{70}$.

995 C. BAC2H analysis of the interactions between T18 and T25 hybrids with PulM and PulG 996 and its derivatives $\mathrm{PulG}^{\mathrm{E} 5 \mathrm{~A}}, \mathrm{PulG}^{\mathrm{T} 2 \mathrm{~A}}$ and $\mathrm{PulG}^{\mathrm{P} 22 \mathrm{~A}} \cdot \beta$-galactosidase activity was measured in at 997 least 6 independent colonies co-transformed with pUT18c and pKT25 plasmid derivatives 998 (Table 1) as described in Materials and Methods. Bar graphs indicate mean values and error 999 bars indicate standard deviation. Statistically significant values relative to the negative control 1000 are indicated above each bar. The difference between certain positive interactions was 1001 assessed for statistical significance and represented by horizontal lines indicating the 1002 compared strains. NC, activity of bacteria producing T18 and T25 as negative control; PC, 1003 activity of positive control strain producing T18-Zip and T25-Zip chimera. The red horizontal 1004 line indicates the background mean $\beta$-galactosidase activity measured in the negative control. $1005 * \mathrm{p}<0.05 ; * * \mathrm{p}<0.01 ; * * * \mathrm{p}<0.0001 ; \mathrm{ns}$, non-significant.

1006 D. Shearing assay of E. coli PAP7460 co-transformed with plasmid pCHAP8184 containing 1007 all pul genes except pulG $(\Delta G)$ complemented with pSU19 (-) or its derivatives carrying pulG 1008 alleles: WT (pCHAP8658), T2A (pCHAP8732) or E5A (pCHAP8663). PulG 1009 immunodetection in $0.05 \mathrm{OD}_{600}$ units of cell and sheared fractions (C, SF). Below, 1010 immunodetection of LamB in the same samples. Lane numbers and Mw markers are depicted.

1011 E. Quantification of the percentage of sheared PulG (mean + s.d.) from three independent 1012 experiments like the one in (D). Ø indicates an empty vector.

1013 F. Secretion assay using E. coli PAP5299 transformed with the same plasmids as in (D). 1014 Immunodetection of PulA in $0.02 \mathrm{OD}_{600}$ units of cell extracts (C) and supernatants (SN) is 1015 shown. $\alpha-R b s B$ is used as a lysis control. Mw markers and lane numbers are shown.

1016 G. Quantification of the percentage of secreted PulA (mean + s.d.) from three independent 1017 assays like the one in $(\mathrm{F}) . \varnothing$ indicates empty vector. 


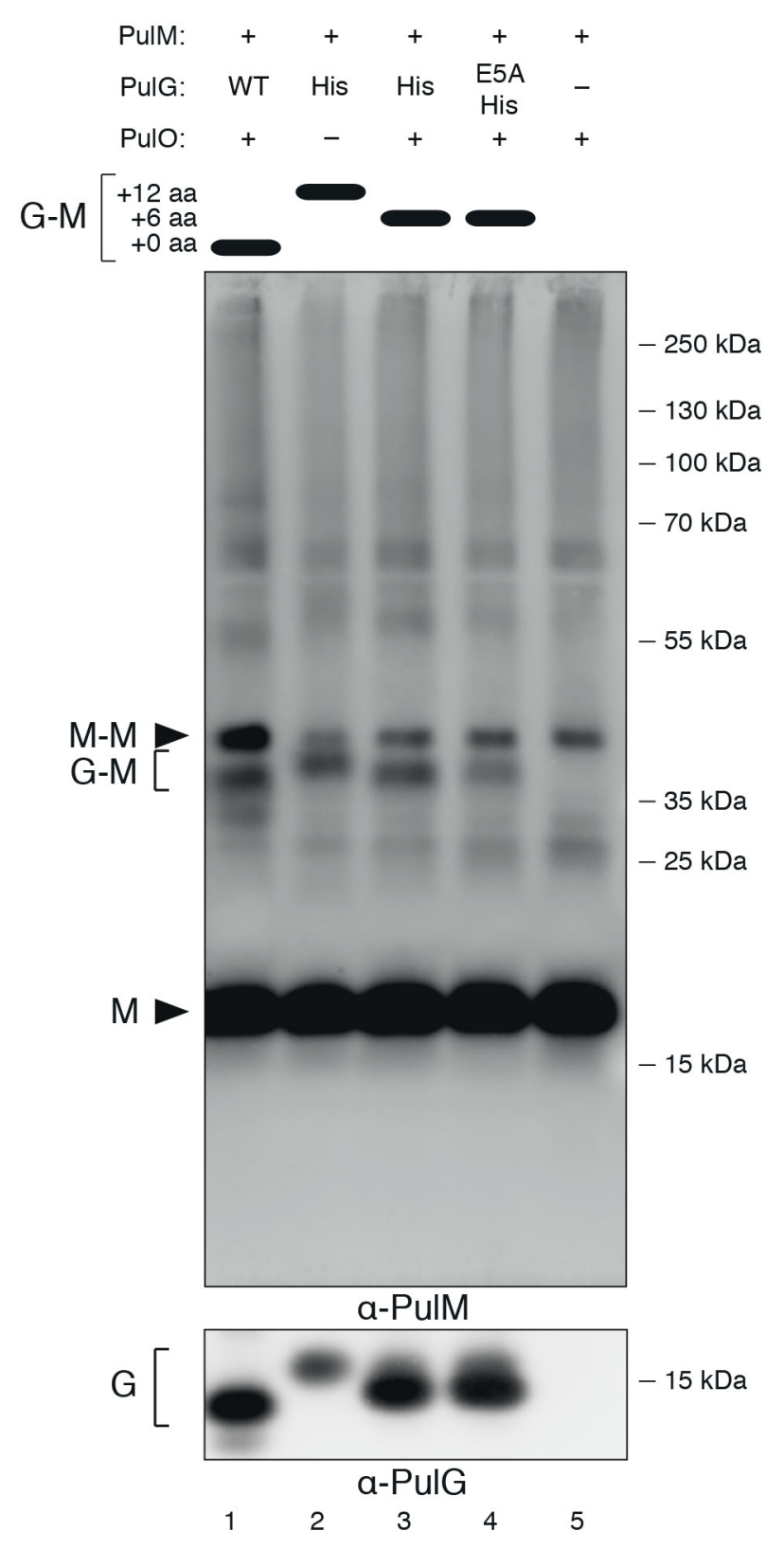

1019 Fig. 6. PulM interacts with PulG in the context of the complete Pul T2SS.

1020 E. coli PAP7460 carrying plasmids encoding the pul genes with either $\Delta p u l G$ (pCHAP8184,

1021 lanes 1 and 3-5) or $\triangle p u l G+\Delta p u l N O$ (pCHAP8400, lane 2) alleles were transformed with 1022 pSU19 or its derivatives encoding PulG (pCHAP8658), PulG-His 6 (pCHAP1362) or PulG ${ }^{\mathrm{E} 5 \mathrm{~A}}$ $1023 \mathrm{His}_{6}$ (pCHAP7785). Total extracts of strains cross-linked in vivo with $0.6 \% \mathrm{FA}$ (Materials and 1024 Methods) analysed by SDS-PAGE and immunodetection with anti-PulM or anti-PulG antisera 1025 are shown. Theoretical relative migration of PulM-PulG heterodimers is depicted according to 1026 the molecular weight of the PulG variant in each case. The positions of the PulM monomers 1027 (M), PulM homodimers (M-M) and the shifting PulG-PulM heterodimers (G-M) are 
1028 indicated. For the $\alpha-\mathrm{PulG}$ blot only the band corresponding to PulG monomer is shown. Mw 1029 markers and lane numbers are indicated. Uncropped images of $\alpha-P u l M$ and $\alpha-P u l G$ Western 1030 blots of untreated and FA-treated samples are shown in Fig. S2.

Initial frame

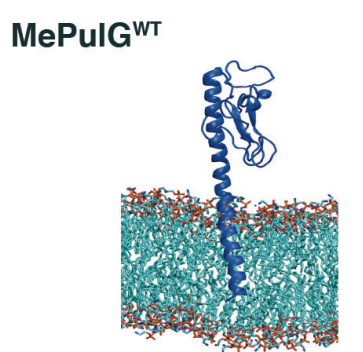

MePulGE5A

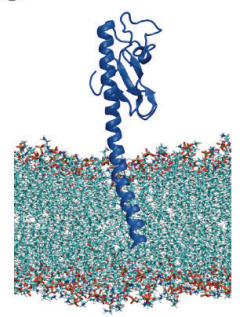

PulGE5A

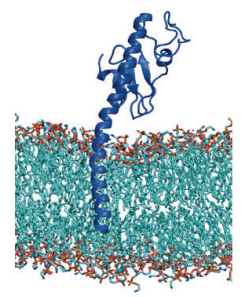

Final frame I
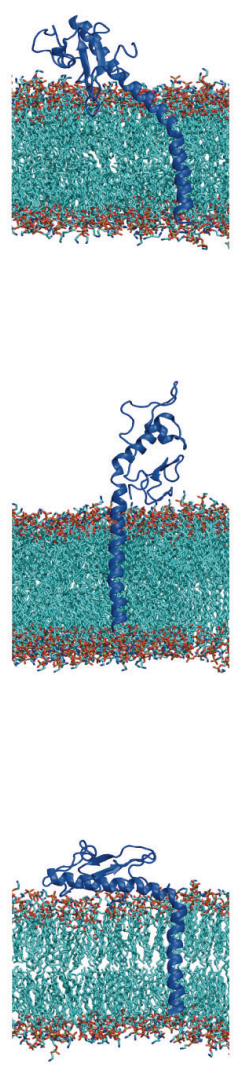

Final frame II
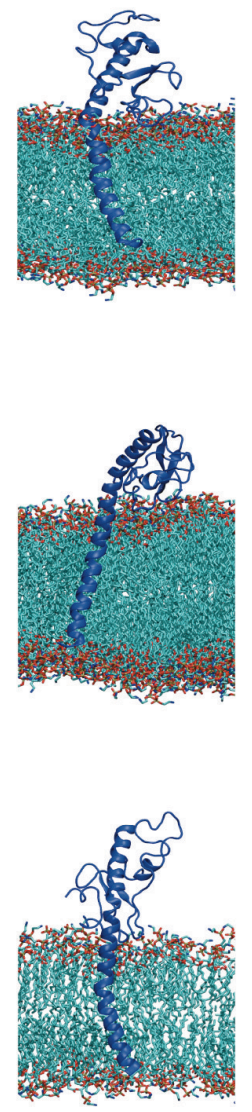

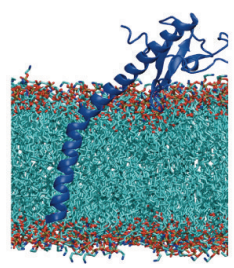

Final frame III
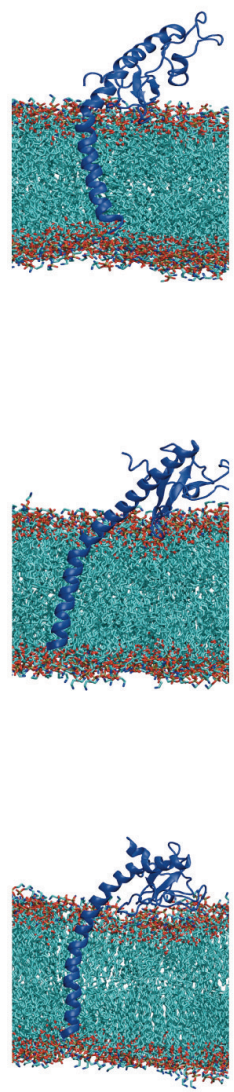

\section{Fig. 7. Visualisation of MD simulations of PulG variants.}

1033 Snapshots of initial and representative final conformations of methylated $\mathrm{MePulG}^{\mathrm{WT}}$, 1034 methylated $\mathrm{MePulG}^{\mathrm{E} 5 \mathrm{~A}}$, and non-methylated $\mathrm{PulG}^{\mathrm{E} 5 \mathrm{~A}}$ variants embedded in a POPE bilayer. 1035 In all the simulations, the protein became more deeply buried, and in all (except PulG ${ }^{\mathrm{E} 5 \mathrm{~A}}$ 1036 replica II) the protein bent towards the membrane surface, allowing the globular domain to 1037 interact with the phospholipids. Changes in F1 and E5 did not cause severe protein 1038 destabilization or membrane perturbation. 
A
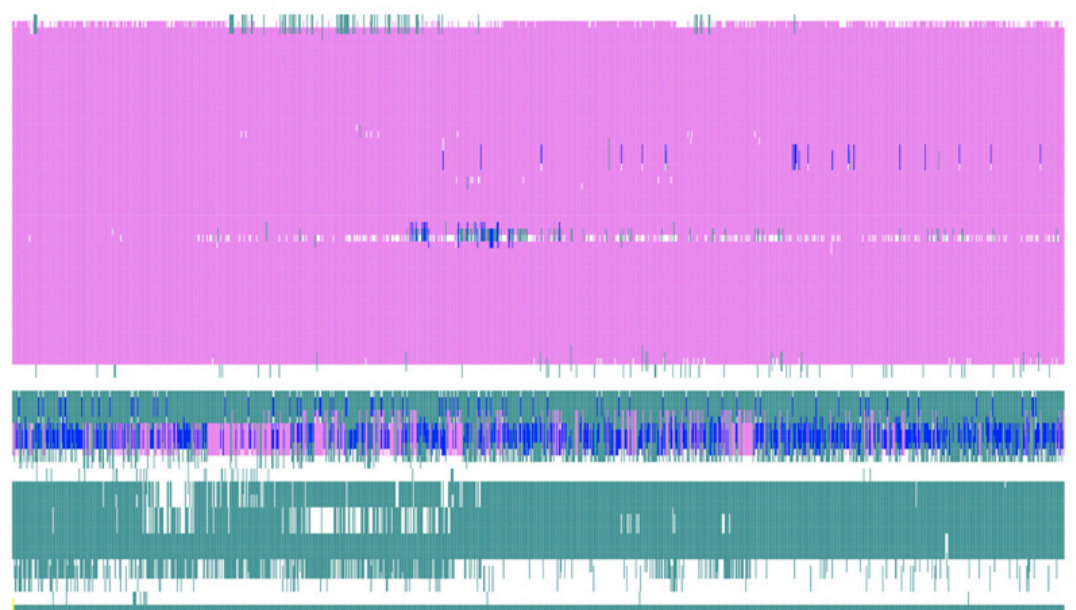

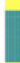

$$
\text { III }
$$

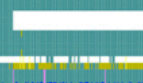

B
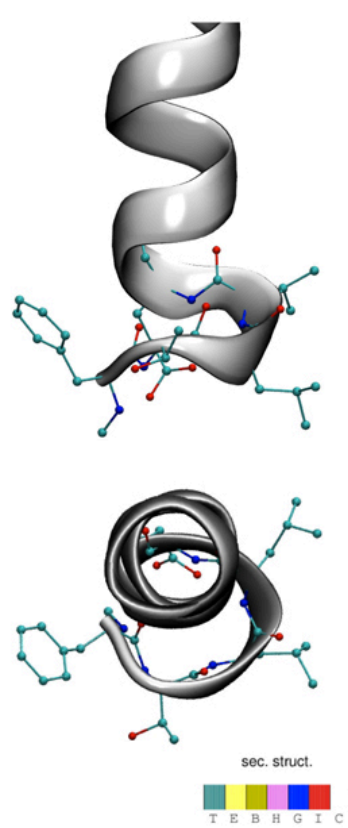

1040 Fig. 8. Secondary structure of PulG during MD simulations.

1041 A. A representative data set showing the maintenance of protein secondary structure during a $1042 \mathrm{MePulG}^{\mathrm{WT}}$ simulation. The graph shows the evolution of the secondary structure of each 1043 residue (y-axis) over time (x-axis), coloured as follows: $\alpha$-helix - purple; 3-10 helix - blue; $\beta$ 1044 sheet - yellow; turn - cyan; coil - white. In $\mathrm{MePulG}^{\mathrm{WT}}$ simulations the first several residues 1045 demonstrated a relaxation of helical structure into either turn or coil structures, suggesting that $1046 \mathrm{MeF} 1$ and E5 destabilized the terminus as they deformed to interact with each other.

1047 B. The MeF1-E5 interaction caused the N-terminus to deform and created a loop, shown here 1048 with the protein backbone in grey and residues 1-5 shown as ball-and-stick structures, 1049 coloured by atom (carbon - cyan, oxygen - red, nitrogen - blue). 
A

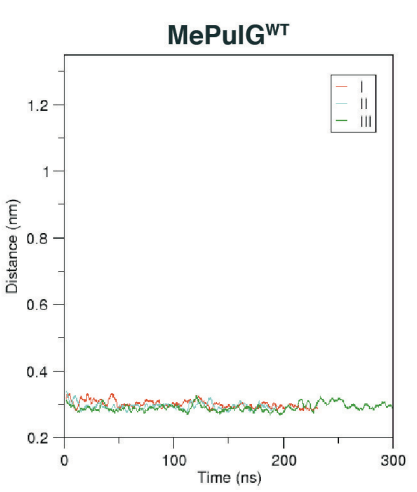

B

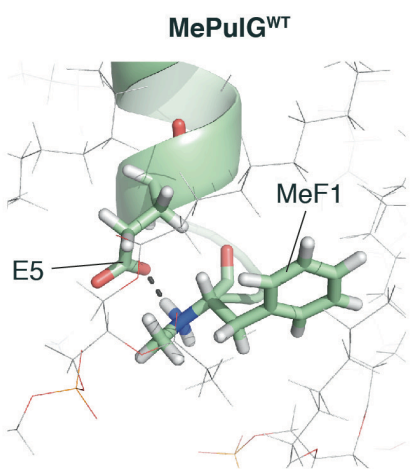

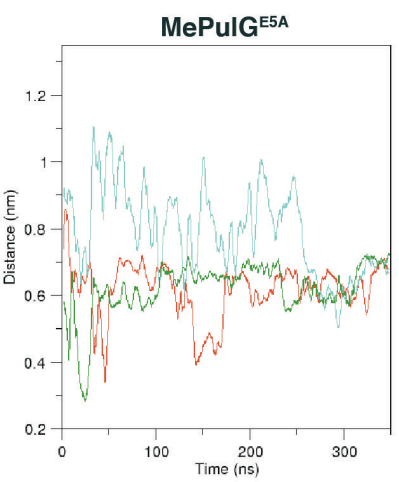
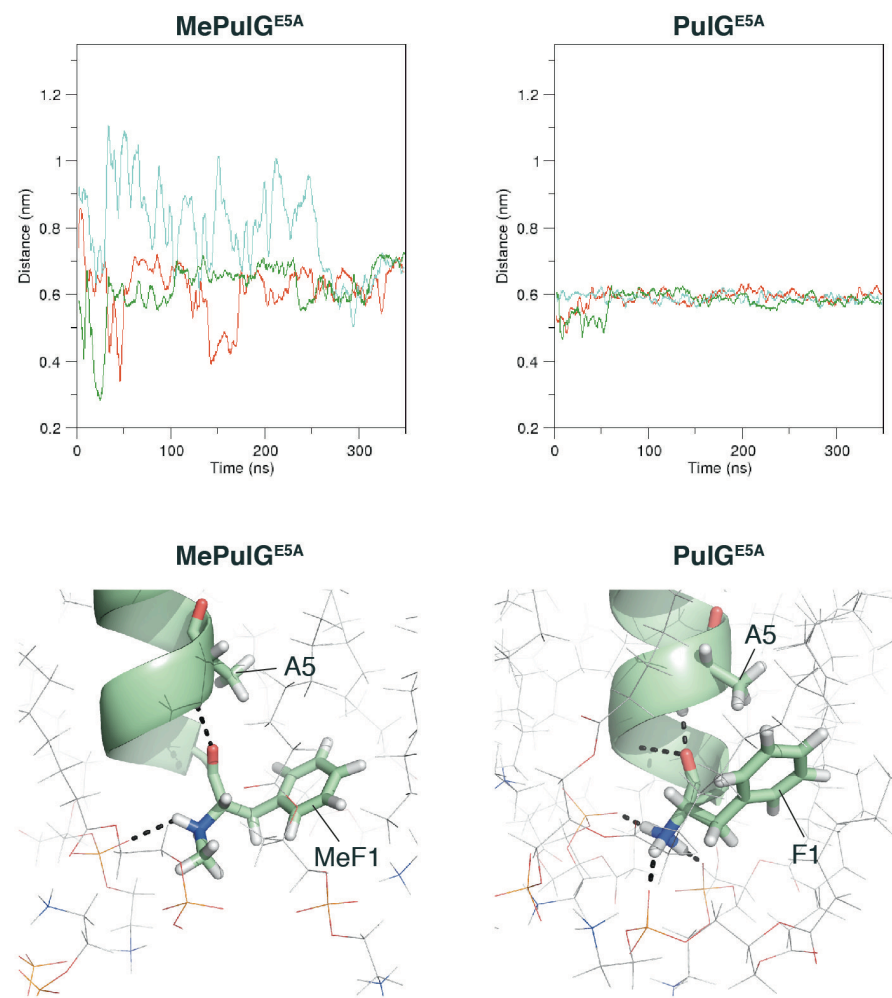

PuIGE5A

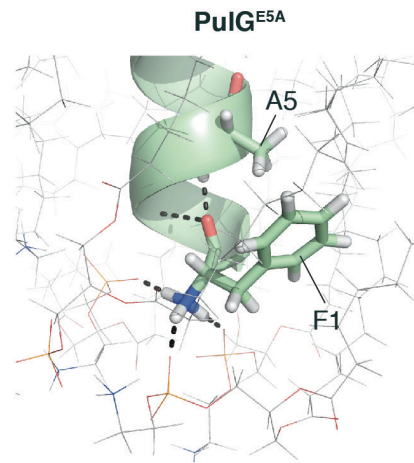

1051 Figure 9. N-terminal intra-molecular and protein-lipid interactions.

1052 A. Graphs showing how the minimum distances between the residue 1 methyl group or amide 1053 terminus with any atom of the residue 5 side-chain varied during the simulations. In the $1054 \mathrm{MePulG}^{\mathrm{WT}}$ system the methyl group of MeF1 and the E5 side-chain remained consistently 1055 within $3 \AA$ of each other, whereas in the mutant systems the distances were much larger. This 1056 suggested that $\mathrm{MePulG}^{\mathrm{WT}}$ E5 promotes intra-molecular interactions with the N-terminal 1057 positive charge of MeF1, anchoring PulG less firmly in the bilayer and priming the protein for 1058 extraction during pseudopilus assembly. In contrast, the N-terminus of the $\mathrm{MePulG}^{\mathrm{E} 5 \mathrm{~A}}$ and $1059 \mathrm{PulG}^{\mathrm{E} 5 \mathrm{~A}}$ mutant variants interact with membrane lipids, anchoring the protein.

1060 B. Representative visualisations of hydrogen bonding of PulG N-terminus to POPE and 1061 solvent; the protein is shown as a green ribbon, with the labelled residues in stick format and 1062 coloured by atom ( $\mathrm{C}$ - green, $\mathrm{O}$ - red, $\mathrm{N}$ - blue, $\mathrm{H}$ - white). Hydrogen bonds are shown as 1063 black dotted lines. POPE is depicted in stick format and coloured by atom ( $\mathrm{C}-$ grey, $\mathrm{O}-\mathrm{red}$, $1064 \mathrm{~N}$ - blue, $\mathrm{H}$ - white, $\mathrm{P}$ - orange). 